\title{
Gene silencing with CRISPRi in bacteria and optimization of dCas9 expression levels
}

Florence DEPARDIEU ${ }^{1}$ and David BIKARD ${ }^{1}$.

${ }^{1}$ Groupe de Biologie de Synthèse, Institut Pasteur, Paris 75015, France.

\section{Corresponding author}

e.mail address : david.bikard@pasteur.fr (D. Bikard)

\section{Highlights}

- Detailed methods and vectors to perform CRISPRi assays in S. aureus and E. coli

- A novel CRISPRi vector based on a very broad host range plasmid

- Methods to optimize the expression level of dcas 9 in a single step to avoid toxicity

\begin{abstract}
The catalytic null mutant of the Cas9 endonuclease from the bacterial CRISPR immune system, known as dCas9, can be guided by a small RNA to bind DNA sequences of interest and block gene transcription in a strategy known as CRISPRi. This powerful gene silencing method has already been used in a large number of species and in high throughput screens. Here we provide detailed design rules, methods and novel vectors to perform CRISPRi experiments in S. aureus and in E. coli, using the well characterized dCas9 protein from S. pyogenes. In particular, we describe a vector based on plasmid pC194 which is broadly used in Firmicutes, as well as a vector based on the very broad host-range rolling circle plasmid pLZ12, reported to replicate in both Firmicutes and Proteobacteria. A potential caveat of adapting dCas 9 tools to various bacterial species is that dCas 9 was shown to be toxic when expressed too strongly. We describe a method to optimize the expression level of dCas9 in order to avoid toxicity while ensuring strong on-target repression activity. We demonstrate this method by optimizing a pLZ12 based vector originally developed for $S$. aureus so that it can work in E. coli. This article should provide all the resources required to perform CRISPRi experiments in a broad range of bacterial species.
\end{abstract}

\section{Keywords}

CRISPRi, dCas9, knockdown, gene silencing, Staphylococcus aureus, Escherichia coli 


\section{Introduction}

Methods to inactivate genes, either through the introduction of mutations or through silencing of gene expression, are critical to the study of gene function. In bacteria, various strategies have been developed to introduce specific mutations through homologous recombination with a DNA template [1,2], but when the targeted genes are critical to the survival of the cell, gene knockouts cannot be obtained. Gene silencing techniques can be inducible, enabling the study of essential genes, and are frequently easier to implement than targeted mutagenesis techniques. Synthetic non coding RNAs can be programmed to alter gene expression through hybridization with messenger RNAs leading to destabilization of the transcript or alteration of translation initiation [3]. Such strategies include: synthetic antisense RNA (asRNA) [4,5,6], toehold switch regulators [7], translation-regulating riboswitches [8], and aptazyme $[9,10]$. In particular asRNA were used to systematically inactivate genes in bacterial genomes and identify essential genes in S. aureus [4] or in Escherichia coli [5,6]. However, native asRNA sytems show structural and mechanistic complexity that makes asRNAs difficult to engineer [11]. Other strategies use non coding RNAs to alter gene expression at the transcription level by modulating transcription termination/attenuation $[12,13]$.

Here we will focus on the inhibition of transcription initiation or elongation which can be achieved by repurposing clustered regularly interspaced short palindromic repeat (CRISPR) systems, in a strategy known as CRISPR interference (CRISPRi) [14,15]. CRISPR systems are adaptive immune systems of prokaryotes, found in most archaea and about $40 \%$ of bacteria [16,17]. Type II systems, and in particular the one from Streptococcus pyogenes, have been the most exploited since they rely on a single protein, Cas9, for target DNA cleavage $[18,19]$. This system consists of an endonuclease Cas9 protein that forms a complex with two RNAs, a mature CRISPR RNA (crRNA) that guides the complex to bind target DNA and a trans-activating crRNA (tracrRNA). The Cas9 endonuclease searches for target sequences by first scanning DNA for the presence of Protospacer Adjacent Motif (PAM) sequences, followed by unwinding of the DNA double helix and hybridization of the crRNA, ultimately leading to a double-strand break at the target position [20]. The crRNA and tracrRNA can be fused to generate a chimeric single guide RNA (sgRNA) that mimics the natural crRNA-tracrRNA [18].

While Cas 9 can be used to introduce mutations at target positions in bacteria, the main outcome of Cas9 cleavage in the chromosome is cell death rather than gene knockout $[21,22,23,24]$. The catalytic dead variant of Cas9 (dCas9) can however be conveniently used to silence gene expression. This variant lacks endonuclease activity due to two mutations (D10A and H841A) inactivating the RuvC and HNH catalytic domains $[14,15,18]$. When dCas9 is coexpressed with a guide RNA, it forms a DNA recognition complex that binds to the specific target, like wild-type Cas9, but without cleavage of the DNA. When bound to promoter sequences, dCas9 can block transcription initiation, and when the guide RNA binds the non-template (coding) strand of a gene, dCas9 attachement is strong enough to block transcription elongation. This strategy, called CRISPRi, can be used to silence genes in bacteria and to repress multiple target genes simultaneously. CRISPR targets are easily programmed by substituting the first 20 nucleotides of the sgRNA sequence to match the nontemplate strand of the target gene, making the design and construction of CRISPR libraries that target specific sets of genes, or the entire genome, straightforward. Genetic screens using CRISPR libraries have already contributed to the identification of gene function and drug modes of action [24,25,26,27].

However, the use of CRISPRi requires a few important design rules. In fact, a recent study in which a pool of guide RNAs was used to target approximately $10^{5}$ positions in the chromosome of 
E. coli, showed that dCas9 can cause off-target effects at positions with as little as $9 \mathrm{nt}$ of homology to the guide RNA [27]. Another observation was the unexpected toxicity of dCas9, when guided by sgRNAs sharing some specific 5 nt PAM-proximal sequences [27]. This phenomenon called the "bad-seed" effect is particularly observed at high dCas9 concentrations, and could be decreased by tuning dCas9 levels while maintaining strong on-target repression. Using such optimized expression cassette enables performing powerful screens using dCas9 [28].

CRISPRi methods have already been adapted to a wide range of bacterial species $[24,25,28,29,30,31,32,33]$. Recent works reported a gene knockdown method in $S$. aureus, using a E. coli-S. aureus shuttle plasmid carrying dCas 9 and a sgRNA [30,34]. These systems can repress the expression of multiple genes simultaneously, silence an entire operon or part of it, and be used for functional studies of essential genes in S. aureus.

In a first part, we detail protocols to perform inducible CRISPRi experiments in S. aureus. This work includes the description of two novel CRISPRi vectors, one based on the commonly used pC194 plasmid [35] and the other based on the broad host range pLZ12 plasmid, a derivative of the streptococcal plasmid pSH71 [36] which is known to replicate in both gram positive and negative bacteria $[36,37,38,39]$. We demonstrate the performance of the strategy by silencing the essential gene $\operatorname{rps} L$. In a second part, we develop a method to optimize the expression level of dCas9 to avoid the toxicity phenomenon observed in E. coli. A library of ribosome binding sites (RBS) controlling the translation of dCas 9 is screened, to select in one step, clones able to survive the expression of dCas 9 in the presence of a bad seed sequence, while maintaining strong on-target repression of a $m$ Cherry reporter gene. This strategy enabled us to quickly adapt the pLZ12 vector initially developed for $S$. aureus to perform CRISPRi assays in E. coli. The "bad-seed" toxicity phenomenon has so far only been reported in E. coli, but is likely present in other bacterial species, where the methods described here can also be applied. Note, however, that the mechanism of the bad seed toxicity is not understood for now and that bad-seed sequences might be different in different species. Further research, and in particular high-throughput screens will be needed to elucidate this point.

\section{Methods to silence genes in $S$. aureus using dCas9}

Two novel vectors carrying the $S$. pyogenes CRISPR-Cas9/dCas9 systems were constructed. The first vector, pFD78, is based on the commonly used pC194 plasmid (Fig. 1) [35]. The other vector, pFD116, is based on the broad host range plasmid pLZ12spec, a spectinomycin resistant variant of plasmid pLZ12 (Fig. 2) [40]. Plasmid pLZ12 was obtained by introducing a multiple cloning site in plasmid pNZ12 [37] which itself derives from the Streptococcus lactis plasmid pSH71 [36,41]. Interestingly, these vectors and their derivatives have been shown to replicate in gram positive bacteria such as Bacillus subtilis [39], Lactobacillus plantarum, Lactobacillus manihotivorans [42], Streptococcus pyogenes [37], Streptococcus pneumoniae [15,39], Streptococcus suis [38], Streptococcus mutans [43], Streptococcus agalactiae [44], as well as gram negative bacteria such as E. coli [39,41,43], Borrelia burgdorferi, Agrobacterium tumefaciens, Acinetobacter baylyi, Francisella novicida [39].

Both vectors carry either cas 9 , or d cas 9 , under the control of an inducible Ptet promoter and a sgRNA controlled constitutively from a promoter of $S$. aureus (Fig. 1 and Fig. 2). In addition, plasmid pFD116 carries an origin of transfer for the RP4 conjugation machinery [45], enabling conjugation to a large number of species (for protocols see references [46] and [47]). We provide all the steps from target choice to guide RNA cloning (Fig. 3) and silencing experiments. Briefly, 
in a first step, the target sequence should be chosen correctly to ensure the presence of a PAM, the proper orientation of dCas9, and to avoid off-target effects. Oligonucleotides can then be designed to allow cloning between two BsaI sites on the vector. Oligonucleotides are phosphorylated and annealed to reprogram the guide carried by the vector using the Golden Gate assembly method [48]. The resulting plasmids can then be introduced in S. aureus, followed by colony PCR and sequencing to check the proper cloning of the guide. Finally, good clones can be used to perform inducible CRISPRi experiments by growing them on a medium with or without anhydrotetracycline (aTc).

\subsection{Plasmids, bacterial strains and culture conditions}

The plasmids used in this study are summarized in Table 1 and the primers to construct them are provided in Table 2. Their construction is described in Fig. 1 and Fig. 2. The strains used in this study are summarized in Table 1. Staphylococcus aureus strain RN4220 [49] was used as host for construction of plasmids based on pC194, while E. coli strain DH5 $\alpha$ was used as host for construction of plasmids based on pLZ12. The strains were grown at $37^{\circ} \mathrm{C}$ in trypticase soy broth (TSB) or on agar (TSA) plates for $S$. aureus and in Luria-Bertani broth or agar for E. coli. Chloramphenicol $(\mathrm{Cm}, 10 \mu \mathrm{g} / \mathrm{ml}$ ) and spectinomycin (Spec, $60 \mu \mathrm{g} / \mathrm{ml}$ in E. coli or $120 \mu \mathrm{g} / \mathrm{ml}$ in S. aureus) was added to the medium to prevent loss of plasmids derived from pC194 and pLZ12, respectively.

\subsection{Choice of the target sequence}

The single guide RNA (sgRNA) used in this study is a chimeric fusion between the crRNA, which encodes a 20-nt target-specific sequence, and the tracrRNA, which enables the formation of active Cas9-guide RNA complex (Fig. 1B) [18]. The target sequence is followed by a PAM sequence ( $5^{\prime}$-NGG-3') which is essential for recognition of target DNA by Cas9 or dCas9.

As an example, we design here guide RNAs targeting the essential rpsL gene, which encodes the ribosomal S12 protein of the 30S subunit of the ribosome and plays a role in translational accuracy (Fig. 3.1).

A few guidelines need to be considered to choose the target sequence / guide.

1. The sequence of the gene should be analyzed to identify a 20 nucleotides target sequence with a proper PAM sequence (5'-NGG-3') present at the 3' of the target (Fig. 3.1).

2. The strand orientation of dCas9 binding does not seem to matter when blocking transcription initiation by targeting a promoter sequence; however when targeting inside a transcribed region, binding of the guide RNA to the non-template (coding) strand (for example, primer F205, Fig. 3.1) is required to block the elongating RNA polymerase [14,15,27].

3. The repression efficiency was initially reported to be inversely correlated with the target distance from the transcription start site by Qi and coallegues [14], but this effect was not observed in a genome-wide screen [27]. Nonetheless, to avoid any risk that a target at the end of the gene might lead to the production of functional truncated proteins, we recommend to choose targets close to the gene start when possible.

4. When the target gene is in an operon, dCas9 will block the expression of all the downstream genes. The effect on upstream genes, also referred to as the reverse-polar effect, seems to depend on the organism. While a strong reverse-polar effect was observed in B. subtilis [25], only a $\sim 50 \%$ reduction in the abundance of upstream transcript was observed in $S$. aureus [30]. The effect also seems weak or non-existing in E. coli for guides targeting more than $100 \mathrm{nt}$ away from the gene end [27]. Note however that the effect in E. coli was measured through the 
fitness defect produced when targeting downstream of essential genes, and not through direct measurement of RNA levels.

5. Candidate target sequences should be checked to ensure the absence of off-target positions. This can simply be achieved by using an alignment search such as NCBI-Blast. dCas9 can tolerate large number of mutations in the PAM-distal part of the guide, but not in the PAMproximal region [50]. Therefore, guides that have candidate off-target positions with a proper PAM and a perfect match with the 10 PAM-proximal bases, should be avoided. Off-target with as little as 9 nucleotides of perfect identity were also reported, but will substantially block gene expression only when present in a promoter sequence, as the interaction is too weak to constitute a strong roadblock for the elongating RNA polymerase. Studies have also shown that dCas9 can bind to non-NGG PAM sites which should be considered when looking for potential offtarget positions [2,51]. Completely avoiding off-targets with such a small sequence requirement might be challenging. Therefore, guides with off-targets that are unlikely to have an effect on the phenotype under study might be considered. Unproblematic off-targets typically include targets on the template strand of genes that are not related to the phenotype under study. To ensure that off-targets are not confounding results, we recommend to study a minimum of three guides per gene.

\subsection{Design of oligonucleotides for cloning in $\operatorname{sgRNA}$}

It is important to note that the PAM needs to be present immediately downstream of the target site in the genomic DNA, but the NGG sequence is not introduced in the sgRNA (Fig. 3.2). To avoid the introduction of extra nucleotides during the assembly of the specific guide sequence in our plasmids, we use the type IIS BsaI restriction enzyme which cleaves outside of its recognition sequence (Fig. 2 and Fig. 3.3). The overhangs produced by BsaI at two sites on the vector have been designed to coincide with the end of the promoter sequence on the left of the guide, and with the sgRNA scaffold region on the right. The left overhang is 5'-TTAG-3' for plasmids based on pC194 (Fig. 3.4) and 5'-ACTA-3' for plasmids based on pLZ12 (Fig. 2), while the right overhang is 5'GTTT-3' for all vectors (Fig. 2 and Fig. 3.4). Thus, the oligonucleotides should be designed to produce the following complementary overhangs when annealed: 5' overhangs of 5'-CTAA-3' and

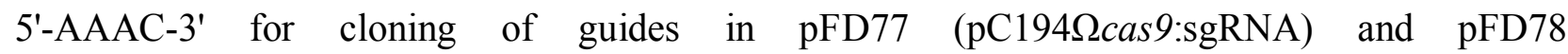

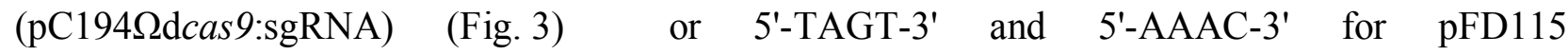

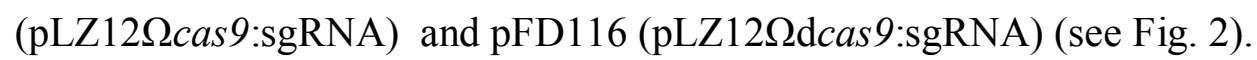

\subsection{Phosphorylation and annealing of oligonucleotides}

Oligonucleotides carrying the guide sequence and the 4nt overhangs necessary for cloning into the vector digested with BsaI (Fig. 3), can be phosphorylated, denaturated and annealed as described below.

\subsubsection{Phosphorylation of oligonucleotides}

Mix in a tube

- $15 \mu \mathrm{l}$ of each oligonucleotide from a $10 \mu \mathrm{M}$ stock

$-14 \mu 1$ of water

- $5 \mu 1$ of T4 ligase buffer 10X with ATP $10 \mathrm{mM}$ (or PNK buffer with addition of ATP at $1 \mathrm{mM}$ final)

- $1 \mu 1$ of T4 PNK (polynucleotide kinase) at 10,000 units/ml (Thermo Scientific)

Incubate at $37^{\circ} \mathrm{C}$ for $30 \mathrm{~min}$ 


\subsubsection{Denaturation of oligonucleotides}

- Add $2.5 \mu 1$ of $\mathrm{NaCl} 1 \mathrm{mM}$

Incubate $5 \mathrm{~min}$ at $95^{\circ} \mathrm{C}$ in a dry heating block.

\subsubsection{Annealing of oligonucleotides}

For proper annealing, temperature needs to be slowly decreased below $40^{\circ} \mathrm{C}$. This can be conveniently achieved by removing the block from the heater and leaving it on the bench.

\subsection{Plasmid extraction from S. aureus $R N 4220$}

Plasmids based on pLZ12 (e.g. pFD116) can be extracted from E. coli using standard kits and protocols. Plasmids based on pC194 (e.g. pFD78) can be isolated from S. aureus RN4220 as follows.

1. Centrifuge 1.5 to $3 \mathrm{ml}$ of an overnight culture in TSB.

2. Resuspend the pellet with $250 \mu 1$ of the lysis solution from a miniprep kit (e.g. buffer A1 of the NucleoSpin kit from Macherey-Nagel, or buffer P1 from Qiagen), and add $4 \mu 1$ of lysostaphin (Ambi Products LLC) at $2 \mathrm{mg} / \mathrm{ml}$.

3. Incubate for $1 \mathrm{~h}$ at $37^{\circ} \mathrm{C}$.

4. Continue the steps of the plasmid purification kit according to the manufacturer's instructions to isolate the plasmid DNA.

\subsection{Cloning of oligonucleotides by Golden Gate method}

Mix of $10 \mu 1$

- $2 \mu 1$ of plasmid DNA

- $2 \mu 1$ of annealed oligonucleotides obtained at step 2.4.3, diluted 1:10 in water

- $1 \mu 1$ of cutsmart buffer 10X (Biolabs)

- $1 \mu 1$ of BsaI-HF (Biolabs)

$-1 \mu 1$ of ATP at $1 \mathrm{mM}$

- $1 \mu 1$ of T4 ligase at 400,000 units/ml (Biolabs, M0202S)

$-2 \mu 1$ of $\mathrm{H}_{2} \mathrm{O}$

Incubate in a thermocycler with the following conditions

- 25 cycles of:

- 3 min at $37^{\circ} \mathrm{C}$

○ 4 min at $16^{\circ} \mathrm{C}$

- $5 \mathrm{~min}$ at $80^{\circ} \mathrm{C}$ to inactivate the enzymes

Dialyze the resulting plasmids on a membrane filter $(0.025 \mu \mathrm{m}$ VSWP, Millipore) for $20 \mathrm{~min}$ at room temperature.

\subsection{Transformation of the assembled plasmid in bacteria.}

Plasmids based on pLZ12 were introduced in E. coli DH5 $\alpha$ using standard transformation procedures described elsewhere [52]. The resulting plasmids were then extracted from E. coli using a miniprep kit and transformed in $S$. aureus RN4220. Plasmids based on pC194 were directly introduced in $S$. aureus strain RN4220 as follows. Plasmids used to transform S. aureus NCTC 8325-4 [53] were extracted from $S$. aureus RN4220 to acquire the proper methylation pattern and 
avoid restriction. Note that an alternative is to extract plasmids from E. coli strain IMXXB which was modified for this purpose [54].

\subsubsection{Preparation of S. aureus electrocompetent cells}

1. Resuspend a single colony from a TSA plate in $3 \mathrm{ml}$ of TSB and incubate at $37^{\circ} \mathrm{C}$ overnight with shaking at $120 \mathrm{rpm}$.

2. Dilute the overnight culture $1: 100$ in $100 \mathrm{ml}$ of TSB in a $250 \mathrm{ml}$ flask.

3. Incubate at $37^{\circ} \mathrm{C}$ with shaking at $120 \mathrm{rpm}$ until an optical density at $600 \mathrm{~nm}$ (OD600) of 0.5-0.6.

4. Transfer the culture in 2 conic bottom tubes of $50 \mathrm{ml}$.

5. Centrifuge the cells at $4,000 \mathrm{~g}$ for $10 \mathrm{~min}$ at $4^{\circ} \mathrm{C}$.

6. Resuspend the pellet in $50 \mathrm{ml}$ of sterile cold-water.

7. Centrifuge and resuspend in $25 \mathrm{ml}$ of sterile cold-water.

8. Repeat step 5 and resuspend in $12.5 \mathrm{ml}$ of sterile cold-water containing $10 \%$ of glycerol.

9. Finally, repeat step 5 and resuspend the cells in $1 \mathrm{ml}$ of sterile cold-water containing $10 \%$ of glycerol.

10. Distribute $100 \mu \mathrm{l}$ aliquots in $1.5 \mathrm{ml}$ Eppendorf tubes, and store at $-80^{\circ} \mathrm{C}$.

\subsubsection{Transformation in S. aureus}

After dialysis, S. aureus strains (RN4220 or 8325-4) were transformed by electroporation with the resulting plasmids.

1. Add $5 \mu 1$ to $10 \mu 1$ of plasmid DNA to $50 \mu 1$ of $S$. aureus electrocompetent cells.

2. Mix and incubate for $10 \mathrm{~min}$ at room temperature.

3. Place the mix in a $0.2 \mathrm{~cm}$ electroporation cuvette

4. Perform the electroporation with the following settings: resistance, $100 \Omega$; capacity, $25 \mu \mathrm{F}$; voltage, $2.5 \mathrm{kV}$.

5. Add $800 \mu \mathrm{l}$ of TSB to the cuvette after electroporation and transfer into $1.5 \mathrm{ml}$ Eppendorf tube.

6. Incubate for $1 \mathrm{~h}$ at $37^{\circ} \mathrm{C}$ with shaking at $120 \mathrm{rpm}$.

7. Spread $50 \mu 1,200 \mu 1$ and the remaining on TSB plates containing antibiotics for selection of the plasmid.

\subsection{Screening of clones by colony PCR in S. aureus}

To check for successful cloning of guide sequences, we perform, in parallel, two PCRs on colony lysates. The first PCR enables to identify clones carrying the guide sequence and the second PCR to amplify a fragment which can be used to confirm the guide sequence by Sanger sequencing. We typically obtain six good clones out of eight screened colonies for the first PCR, and Sanger sequencing results show a mutation only once every ten screened clones on average.

\subsubsection{Preparation of colony PCR buffer}

Mix for $500 \mathrm{ml}$ :

$-9.32 \mathrm{~g}$ of $\mathrm{KCl}$ (or $125 \mathrm{ml}$ of $\mathrm{KCl} 1 \mathrm{M}$ )

- $0.24 \mathrm{~g}$ of $\mathrm{MgCl}_{2}$ (or $2.5 \mathrm{ml}$ of $\mathrm{MgCl}_{2} 1 \mathrm{M}$ )

$-25 \mathrm{ml}$ of Tris- $\mathrm{HCl} \mathrm{pH} 9.0$ at $1 \mathrm{M}$

$-2.5 \mathrm{ml}$ of Triton $\mathrm{X}-100$

Add $\mathrm{H}_{2} \mathrm{O}$ qsp $500 \mathrm{ml}$ 


\subsubsection{Colony PCR for S. aureus}

1. Pick one colony and resuspend it in $40 \mu 1$ of colony PCR buffer.

2. Add $2 \mu 1$ of lysostaphin at $2 \mathrm{mg} / \mathrm{ml}$.

3. Execute a lysis program in a thermocycler: $20 \mathrm{~min}$ at $37^{\circ} \mathrm{C}$ following by $10 \mathrm{~min}$ at $98^{\circ} \mathrm{C}$ and cool down to $10^{\circ} \mathrm{C}$.

4. Spin down the tubes for $3 \mathrm{~min}$ at $13,000 \mathrm{rpm}$.

5. Use 2 to $3 \mu 1$ of the lysate as template DNA for your PCR reaction.

\subsubsection{Amplification by PCR}

1. Perform a first PCR using the reverse primer of the guide and a forward primer which anneals upstream of the guide to amplify a fragment with a correct size (between $300 \mathrm{bp}$ and $1 \mathrm{~kb}$ ).

For example, here we chose a forward primer annealing to the end of dcas9 (LC547) to obtain a fragment with an expected size of $384 \mathrm{bp}$.

2. In parallel, perform a second PCR, with primers, surrounding the guide region (we used here LC547/F191 for plasmids derived from pC194 or LC547/AB269 for plasmids derived from pLZ12).

3. Purify the second PCR, using a PCR clean up kit, for clones where the first PCR was positive, and send it for Sanger sequencing.

\subsection{Test of knockdown efficiency by cas 9 or dcas 9 induction on agar plates}

Control strains carrying either an empty vector or a sgRNA without target, and their derivatives harboring specific sgRNAs were grown overnight in TSB with appropriate antibiotic $(\mathrm{Cm} 10 \mu \mathrm{g} / \mathrm{ml}$ for $\mathrm{pC} 194$ and Spec $120 \mu \mathrm{g} / \mathrm{ml}$ for pLZ12), followed by serial dilutions and $5 \mu 1$ were spotted on TSA with or without aTc at $0.025 \mu \mathrm{g} / \mathrm{ml}$ for the plasmids derived from pC194 or at $0.5 \mu \mathrm{g} / \mathrm{ml}$ for the plasmids derived from pLZ12.

\section{Analysis of knockdown efficiency for the essential gene, rpsL, in $S$. aureus}

We constructed plasmids carrying d cas 9 to perform CRISPRi assays. The identical plasmids carrying cas 9 were also constructed as controls and to demonstrate the consequences of Cas 9 cleavage in the chromosome of $S$. aureus. The cas 9 or dcas 9 genes are controlled by an aTcinducible Ptet promoter, whereas the sgRNA is controlled constitutively from the PpflB promoter of $S$. aureus [55]. The Cas9-sgRNA or dCas9-sgRNA expression cassettes were introduced either on plasmid pC194 (Fig. 1) or on the broad host range plasmid pLZ12spec (Fig. 2), yielding plasmids

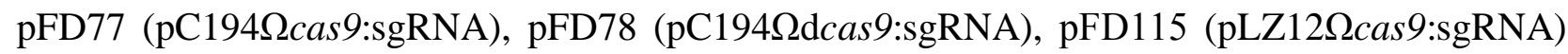

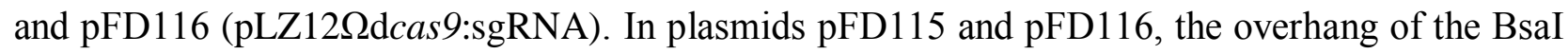
site on the promoter side was changed to make it compatible with the previously published plasmid psgRNA (Fig. 2) [27]. The BsaI guide was used as non-targeting negative control since it has no specific target in $S$. aureus or in E. coli.

To measure the ability of dCas9 to repress the essential $r p s L$ gene of $S$. aureus, guides were designed to target the template strand (T-rpsL) or the coding strand (C-rpsL) (Table 3). These guides

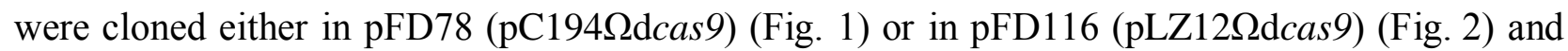
introduced into $S$. aureus RN4220 as detailed in Method section 2 and in Fig. 3. The primers used for the guides are reported in Table 3. Variants of these plasmids with cas 9 instead of d cas 9 were also tested and are presented in Table 3. 
S. aureus RN4220 carrying the plasmids described above or an empty control vector were grown overnight followed by serial dilutions and plating in the absence or in the presence of aTc (Fig. 4). In the absence of aTc, none of the strains showed a growth defect. As expected, with dcas 9 and in the presence of aTc, sgRNAs targeting rpsL on the coding strand strongly inhibited growth on agar plates. The guide targeting the template strand showed a moderate effect for the $\mathrm{pC} 194$ based vector (pFD78) (Fig. 4A). This is consistent with the fact that binding in that orientation only partially represses gene expression [14,15,27]. This phenomenon was less striking for the pLZ12 based vector (pFD116), suggesting that dCas9 is expressed at a lower level in this vector (Fig. 4B). In the presence of the Cas9 nuclease, both the sgRNA targeting the template or coding strands of rpsL led to efficient cell death (Fig. 4C and D), consistently with the fact that Cas9 cleave both DNA strands regardless of orientation. In this case, cell death is due to the destruction of the host DNA rather than silencing of the $\operatorname{rps} L$ gene [23].

We also tested here whether the toxicity phenomenon caused by specific seed sequences previously described in E. coli would also occur in S. aureus (Fig. 4B). To this end, guides that do not show any target in $S$. aureus but carry two of the strongest bad seeds (ACCCA and AGGAA) for $E$. coli were cloned in plasmid pFD116. These guides showed no toxicity in S. aureus suggesting that the bad seed effect either does not exist in $S$. aureus or that it occurs with other seed sequences that are yet to be identified.

Finally, the same plasmids were electroporated in S. aureus 8325-4 [53] and knockdown efficiency measured in the same way (Fig. 5). The results (Fig. 5) were similar to those obtained with S. aureus RN4220 (Fig. 4). Altogether these results demonstrate efficient and inducible

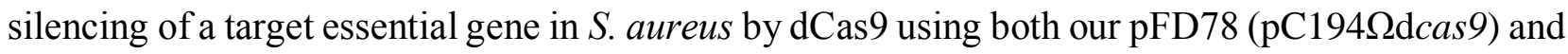

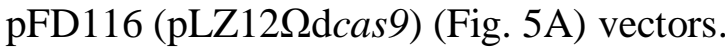

\section{Method to optimize the expression level of dcas 9 in $E$. coli}

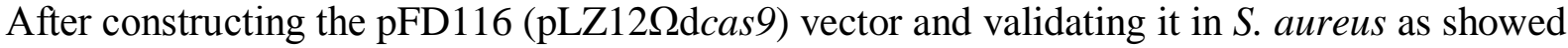
in Fig. 4B and Fig. 5A, we observed that expression of dCas9 from this plasmid in E. coli is highly toxic, presumably due to expression levels that are too high. We first attempted to reduce the concentration of aTc in order to obtain an intermediate level of dCas9 expression. However, this proved to be challenging as a concentration of $0.025 \mu \mathrm{g} / \mathrm{ml}$ of aTc did not lead to a dCas 9 level high enough to strongly block the expression of a target mcherry, while a concentration of $0.05 \mu \mathrm{g} / \mathrm{ml}$ was already too high and toxic. This is likely due to a sharp response of the Ptet promoter in this concentration range and to the fact that in a regime of low transcription and high translation the amount of dCas9 protein might vary widely depending on the number of transcripts available in the cell $[56,57]$. In order to obtain a more stable expression level, a high transcription rate and lower translation rate are desirable.

We thus developed a method to select, in a single step, dCas9 expression vectors with an optimized RBS able to avoid the bad seed toxicity phenomenon while maintaining strong on-target repression (Fig. 6). In E. coli, the $y h h X$ gene encodes a putative oxidoreductase and is not an essential gene. However, we previously reported that at high dCas9 concentrations, a guide targeting $y h h X$ on the template strand (T-yhhX) and carrying an ACCCA PAM-proximal sequence was highly toxic in E. coli [27]. In fact, all guides sharing this ACCCA seed sequence, as well as all guides sharing seed sequences from a set of $\sim 100$ such 5 nt sequences, are toxic to $E$. coli when dCas 9 is highly expressed regardless of their actual target sequence. This toxicity phenomenon can be alleviated by reducing the expression level of dCas 9 while maintaining a sufficiently high dCas 9 
concentration to strongly block the expression of target genes. To easily fine-tune dCas 9 expression level, we screened RBS variants in E. coli strain FD1, a derivative of MG1655, which we modified to carry the $y h h X$ target sequence in front of a constitutively expressed $m$ Cherry reporter gene integrated in the chromosome (see 4.1.2 and Fig. 6). This strain allows, in one step, the screening of clones able to survive the expression of dCas9 in the presence of a bad seed sequence, while still efficiently silencing the expression of mCherry.

\subsection{Plasmids, bacterial strains and culture conditions.}

Plasmids and bacterial strains are summarized in Table 1.

\subsubsection{Plasmid pFD119 (pLZ12Sdcas9:T-yhhX).}

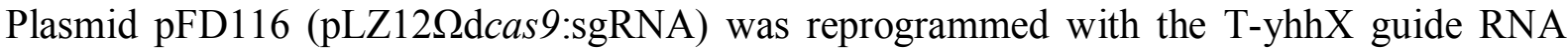
using oligonucleotides LC975/LC976 as described in Method section 2.4 to 2.8, giving plasmid pFD119 (pLZ12 $\Omega$ dcas9:T-yhhX ACCCA) (Fig. 6.1). E. coli DH5 $\alpha$ (New England Biolabs) was used as host for the construction of plasmids based on pLZ12 [40] and was grown in Luria-Bertani (LB) broth or agar at $37^{\circ} \mathrm{C}$. To prevent loss of plasmids derived from pLZ12, spectinomycin $(60 \mu \mathrm{g} / \mathrm{ml})$ was added to the medium.

\subsubsection{Construction of the E. coli screening strain FD1.}

Strain FD1 can be obtained from addgene (\#125258). This strain was constructed through integration of plasmid pFD145 at the phage lambda attB site (Tables 1 and 2 and Fig. 6.2). Plasmid pFD145 is an integrative vector based on pOSIP-KL (KmR, \#45984, Addgene) which carries the yhhX target sequence followed by a mCherry reporter gene (Fig. 6.2). The yhhX target was introduced on the non-template strand between a constitutive promoter and the $m$ Cherry reporter gene through PCR amplification of plasmid pOSIP-KL $\Omega$ mCherry (pAV13) with primers F306/F299, followed by Gibson assembly [27,58]. E. coli E4644 (TransforMax ${ }^{\mathrm{TM}} \mathrm{EC}$ 100D ${ }^{\mathrm{TM}}$ pir+, Lucigen) was used as host for recombinant plasmids based on pOSIP-KL and was grown at $30^{\circ} \mathrm{C}$ in LB broth or agar supplemented with kanamycin $50 \mu \mathrm{g} / \mathrm{ml}$. Plasmid pFD145 containing the lambda integrase, lambda attP site and a kanamycin-resistance $(\mathrm{KmR})$ cassette was integrated at the lambda attB site in the chromosome of $E$. coli MG1655. The integration was verified by PCR, and the backbone was flipped out using the pE-FLP (AmpR, \#45978, Addgene) plasmid able to recombine FRT sites flanking the backbone as previously described [59]. pE-FLP was then cured through serial restreaks on LB plates, leading to the strain FD1 (Fig. 6.2).

\subsection{Construction of a RBS library and selection of optimal dCas 9 expression levels in E. coli}

\subsubsection{Construction of the RBS library}

Introducing random mutations in RBS sequences is a powerful method to obtain variants that express a given protein at various levels [60]. These mutations can be introduced by PCR using oligonucleotides with random or pseudo-random bases at certain positions. Such oligonucleotides need to be designed specifically depending on the gene of interest and its 5'UTR sequence. Here, a RBS library was generated on plasmid pFD119 by randomizing 9 positions in the sequence upstream of the dcas 9 start codon as follows GAATTCNGANGCATNNNNGNNNCAAAAATG. We arbitrarily targeted positions in the 5'-UTR in GA-rich regions typical of RBS sequences. More rational approaches can also be employed based on RBS strength prediction algorithms [61]. These random bases were synthesized in primers F308 and F309 (Table 2), and were introduced on plasmid pFD119 through two PCRs with primers F219/F308 and F217/F309 of the whole plasmid (Fig. 6.1), 
followed by Gibson assembly. The resulting plasmids were introduced into competent FD1 cells by heat shock at $42^{\circ} \mathrm{C}$ (Fig. 6.2), following standard protocols [52], and plated on LB agar supplemented with $60 \mu \mathrm{g} / \mathrm{ml}$ spectinomycin and $0.5 \mu \mathrm{g} / \mathrm{ml}$ aTc to induce the expression of dCas9.

\subsubsection{Selection of clones with non-toxic expression levels of $d$ Cas 9 and good target repression}

All clones that grow on the transformation plates are able to survive dCas 9 expression in the presence of a bad seed sequence (Fig. 6.3).

1. Take a picture of the plate with a fluorescence imager (mCherry excitation wavelength is $587 \mathrm{~nm}$ and emission $610 \mathrm{~nm}$ ). Select large colonies whose fluorescence intensity appears the lowest (Fig. 6.3). These clones are able to grow well in the presence of the T-yhhX (ACCCA) guide while still efficiently blocking the expression of mCherry. To evaluate these clones more thoroughly, go through the following steps.

2. Resuspend clones in $50 \mu 1 \mathrm{PBS}$ and perform serial dilutions from undiluted to $10^{-7}$.

3. Plate spots of $5 \mu 1$ on LB agar in the absence or presence of aTc (Fig. 6.4) supplemented with the plasmid selection antibiotic. On the same plate, spot dilutions of the control strain carrying the empty vector. Incubate at $37^{\circ} \mathrm{C}$ overnight.

4. Compare colony size of the selected clones with the control on plates with or without inducer (Fig. 6.4). The clones should grow as well in the presence of aTc as in the absence of aTc.

5. Take a picture with a fluorescence imager to select the clones that grow best in the presence of aTc while showing a decreased level of mCherry expression (Fig. 6.4).

\subsubsection{Measurement of repression efficiency with a spectrophotometer}

Finally, to obtain a more quantitative measurement of growth and repression efficiency of selected clones, measurements can be achieved in a spectrophotometer (Fig. 6.5). We used here an Infinite M200 PRO reader (TECAN).

1. Grow the clones overnight in LB supplemented with antibiotics and aTc at $0.5 \mu \mathrm{g} / \mathrm{ml}$ at $37^{\circ} \mathrm{C}$ with shaking.

2. Dilute 1:100 the overnight culture in 200ul LB supplemented with antibiotics and aTc at $0.5 \mu \mathrm{g} / \mathrm{ml}$ in a 96 well black polystyrene plate with clear flat bottom (e.g. Corning \#3603).

3. Incubate at $37^{\circ} \mathrm{C}$ in the spectrophotometer with shaking during $12 \mathrm{~h}$.

4. For growth curves, absorbance was measured every $5 \mathrm{~min}$ at $600 \mathrm{~nm}$.

5. mCherry fluorescence was measured every $5 \mathrm{~min}$ at $582 \mathrm{~nm}$ for excitation and at $615 \mathrm{~nm}$ for emission for each clone with and without induction.

6. Select clones that grow best in the presence of aTc, while showing a decreased level of mCherry expression.

\subsubsection{Sequencing of $R B S$}

1. Sequence the selected RBS by Sanger sequencing of the corresponding region.

2. Compare the results with the original RBS to see the modifications (Fig. 6.6).

\section{Optimization of plasmid pFD116 to perform CRISPRi experiments in E. coli}




\subsection{Results of the optimisation of the RBS library}

A RBS library (Table 2) was cloned on plasmid pFD116 programmed with the T-yhhX guide RNA as described above. Strain FD1 was transformed with this resulting library (Fig. 6). Among all the clones that grew on the plates with aTc after transformation, eighteen clones were selected, for their ability to survive dCas9 expression in the presence of a bad seed sequence, while still efficiently blocking the expression of mCherry when guided by the T-yhhX sgRNA (Fig. 6.3). Serial dilutions were performed directly from these clones and plated on LB in the absence or in the presence of aTc and the mCherry expression was analyzed with an Azure Biosystem apparatus (Fig. 6.4). The results show that after induction, only 10 out of 18 clones were able to grow as well as the control strain while repressing mCherry (Fig. 6.5). These ten clones were sequenced to compare their RBS with the strong RBS of pFD116 (Fig. 6.6). Altogether these results show that the dCas9 expression level can be easily tuned to decrease the bad seed effect while maintaining good on-target repression in a single step experiment. Note that only a single bad seed sequence was tested here. However, we previously showed that reducing the expression level of dCas9 alleviates the toxicity of all bad seeds in a similar manner [27].

\subsection{Silencing the E. coli rpsL gene with optimized pFD116 vectors}

After this screening process, we obtain optimized dcas 9 vectors which contain the T-yhhX guide RNA, and which cannot be easily reprogrammed with a different guide. To obtain a reprogrammable plasmid, three of the selected RBS (clone number 4, 5 and 7, Fig. 6.6) were introduced in plasmid pFD116 through PCR followed by Gibson assembly to generate plasmids pFD152 (RBS-4), pFD153 (RBS-5) and pFD154 (RBS-7) (Table 1). Primers used are provided in Table 2.

To validate the plasmids obtained, we programmed them to target rpsL. Guide RNAs targeting the $\operatorname{rps} L$ gene of $S$. aureus or E. coli were designed to bind the template strand or the coding strand (Table 3). E. coli cells carrying these plasmids were grown overnight followed by serial dilutions and plating in the absence or presence of aTc (Fig. 7). In the absence of aTc, none of the strains showed a growth defect. In the presence of aTc, only the sgRNA targeting the rpsL gene of $E$. coli on the coding strand showed a decreased plating efficiency, whereas all the other strains were able to grow normally. The same results were obtained for all three optimized RBS tested. As expected, guides programmed to target the S. aureus rpsL gene had no effect on E. coli (Fig. 7).

\subsection{Performance of pFD116 and pFD152 as a function of aTc concentration}

Finally, we evaluated the ability of pFD116 and pFD152 to block the expression of a target mCherry gene without toxicity as a function of aTc concentration. To this end the mCherry reporter gene was cloned on the plasmids allowing in future work to measure the performance of these plasmids in various strains. A guide RNA was introduced in the resulting plasmids to target the coding strand of mCherry and to block transcription elongation. In the absence of aTc, the plasmid derived from pFD116, which was not optimized, led to a basal repression of the mCherry target of $16 \%$ without induction, while the plasmid derived from the optimized pFD152 led to a basal repression level of $8 \%$, likely due to a small leakage of dCas9 expression from the Ptet (Fig. 8).

Without RBS optimization (pFD116) a concentration of $0.025 \mu \mathrm{g} / \mathrm{ml}$ of aTc led to dCas9 levels too low to see a strong repression of the target gene, while at $0.05 \mu \mathrm{g} / \mathrm{ml}$ of aTc, dCas 9 levels are already too high and toxic (Fig.8). This provides a very small range of concentration to work with, especially considering the fact that aTc is not a very stable molecule and is light sensitive. In the 
case of the optimized vector derived from pFD152, a strong repression of the target was obtained from a concentration of $0.5 \mu \mathrm{g} / \mathrm{ml}$ to the maximal concentration tested of $2 \mu \mathrm{g} / \mathrm{ml}$, with only mild toxicity (reduction in colony size compared to the control) starting at $1.5 \mu \mathrm{g} / \mathrm{ml}$ (Fig.8). The optimized pFD152 therefore provides more robust expression system than pFD116. We speculate that when using pFD116 with low aTc concentrations, dCas9 is expressed from a small number of transcripts but with a high translation initiation rate. This can lead to high fluctuations in the expression level after transcription events. Noisy expression of dCas9 might be responsible for the toxicity and low robustness observed [56,57]. Previous studies have also shown how synthetic gene constructs with strong RBS tend to impose a larger burden on the cell, which could also explain the best performance of pFD152 [62].

\section{Concluding remarks}

We provide simple yet powerful methods, to control gene expression in bacteria by using dCas9 to block transcription initiation or elongation. The vectors described here are easy to reprogram and use in various contexts. The pC194 based vector pFD78 should work in many Firmicutes without further modifications. Our novel pLZ12 vector works, in both $S$. aureus and E. coli, but with the requirement to adapt the RBS to obtain suitable expression levels in each species for which we provide methods here. This same vector should also be functional in a broad range of other bacterial species, where it might be necessary to optimize dCas 9 expression levels in a similar fashion. While the CRISPRi strategy has now already been employed in several bacterial species, this was done through the tedious development of specific vectors by many groups independently. To address this issue, Peters and colleagues, recently, developed "Mobile-CRISPRi" to provide a suite of CRISPRi vectors that combines modularity, stable genomic integration, and ease of transfer to diverse bacteria by conjugation [47]. Two systems were constructed: one for Gram negative bacteria based on the Tn7 transposition system, and the other for Gram positive bacteria (B. subtilis, S. aureus, E. faecalis and Listeria monocytogenes) using an integrative and conjugative element of Bacillus subtilis (ICEBs 1). We now extend the toolbox of CRISPRi tools that can be easily used in a wide range of bacterial species, by providing plasmids that are functional in both gram positive and negative bacteria. Note that the use of plasmids rather than integrative elements means that there is no restriction to use only strains where the attachment site of the integrative element is present.

\section{Acknowledgments}

We thank F. Rousset for helpful discussion.

\section{Funding sources}

This work was supported by the European Research Council (ERC) under the Europe Union's Horizon 2020 research and innovation program (grant agreement No [677823]), by the French Government's Investissement d'Avenir program and by Laboratoire d'Excellence 'Integrative Biology of Emerging Infectious Diseases' (ANR-10-LABX-62-IBEID), and by the PasteurWeizmann consortium. 


\section{Figure Legends}

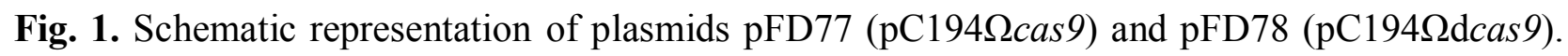
(A) Map and construction process of the plasmids. The Ptet promoter inducible by anhydrotetracycline was amplified with primers F190/F139 from pKL55-iTet-RC12 [22]. The cas 9 or dcas 9 genes were amplified from pDB114 [22] for cas 9 or from pDB182 (a dCas9 variant of pDB114) for d cas 9 using primers F141/B295. A 533-bp synthetic DNA fragment (gBlocks gene fragment from IDT) containing a strong transcription terminator (BioBrick part BBa_B0015), the PpflB promoter [55], and a sgRNA composed of a 5' variable region (guide) with two BsaI sites and the constant region (scaffold), was amplified using primers LC547/F191. These three fragments were assembled by Gibson method in pC194 amplified with primers F188/F189 (Table 2) to generate plasmids pFD77 and pFD78. (B) Sequence of the synthetic DNA fragment.

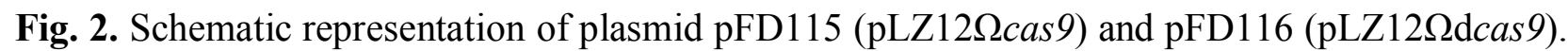
The DNA region containing cas 9 or d cas 9 gene under the control of Ptet promoter, the transcription terminator and the sgRNA under the control of the constitutive promoter PpflB was cloned on pFD110, a variant of pLZ12spec with a RP4 origin of transfer (Tables 1 and 2). In a second step, one of the two BsaI sites used to clone the guides was modified (see primers F236/F237, Table 2) to be identical to the one of psgRNA [27] and enable the reuse of oligonucleotides.

Fig. 3. Target selection and guide RNA cloning. As an illustration, a target position is selected on the non-template (coding) strand of the $r p s L$ gene from $S$. aureus. NGG PAM sequences are highlighted in red.

Fig. 4. Silencing vs. cleavage of $r p s L$ with dCas9 or Cas9 in S. aureus RN4220. Cells were grown overnight, followed by serial dilutions and plating on chloramphenicol $10 \mu \mathrm{g} / \mathrm{ml}$ (pC194) or on spectinomycin $120 \mu \mathrm{g} / \mathrm{ml}$ (for pLZ12) with induction or not by aTc at the concentration indicated. The effect of empty vector, a non targeting sgRNA control, and the rpsL sgRNAs targeting the template strand (T-rpsL) or the coding strand (C-rpsL) in S. aureus RN4220/pC194 (A) or in RN4220/pLZ12 (B) with dcas9 and in RN4220/pC194 (C) or in RN4220/pLZ12 (D) with cas 9 is presented without and with aTc. Two of the strongest specific bad seeds (ACCCA and AGGAA) in

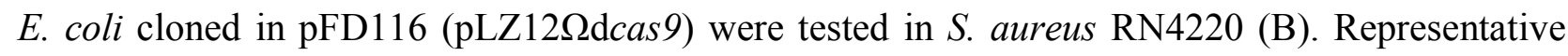
figures from experiments performed in triplicate.

Fig. 5. Silencing vs. cleavage of $r p s L$ with (A) dCas9 or (B) Cas9 in S. aureus 8235-4. Effect of the empty vector, a non targeting sgRNA control, the T-rpsL guide targeting the template strand or the C-rpsL guide targeting the coding strand on plating efficiency in S. aureus 8235-4. Cells were grown overnight, followed by serial dilutions and plating on spectinomycin $120 \mu \mathrm{g} / \mathrm{ml}$ with induction or not by aTc at $0.5 \mu \mathrm{g} / \mathrm{ml}$. Representative figures from three independent experiments.

Fig. 6. Method to fine tune the expression level of dCas9 to eliminate toxicity while maintaining strong on-target repression.

Step 1. An RBS library was introduced by Gibson assembly in plasmid pFD119 carrying dcas 9 inducible by Ptet and the T-yhhX guide RNA carrying a « bad seed » sequence (ACCCA) expressed constitutively from PpflB. Step 2. The resulting plasmid was transformed in strain FD1 harboring the $\mathrm{T}$-yhhX target sequence upstream from a $m$ Cherry reporter gene. Step 3. The transformation reaction was plated on LB agar supplemented with spectinomycin $60 \mu \mathrm{g} / \mathrm{ml}$ and aTc $0.5 \mu \mathrm{g} / \mathrm{ml}$. Clones forming large colonies and showing a low fluorescence signal were selected. Step 4. To confirm the phenotype of these clones, they were serialy diluted and spotted on LB + Spec $60 \mu \mathrm{g} / \mathrm{ml}+\mathrm{aTc}$ 
$0.5 \mu \mathrm{g} / \mathrm{ml}$, in parallel with cells carrying an empty vector control or plasmid pFD119. Clones forming colony of normal size and which strongly repressed mCherry were selected for further analysis. Step 5. The clones were grown in triplicate overnight at $37^{\circ} \mathrm{C}$ in the presence of antibiotic ( $\mathrm{Spec} 60 \mu \mathrm{g} / \mathrm{ml}$ ) and aTc $0.5 \mu \mathrm{g} / \mathrm{ml}$, and fluorescence measured in a Infinite M200 PRO reader (TECAN) at $582 \mathrm{~nm}$ for excitation and at $615 \mathrm{~nm}$ for emission. Fluorescence/OD values, normalized to the level of the FD1 control are reported as the mean values and the error bars reflect the standard deviation from the mean. Step 6. Selected clones were sequenced from plasmid DNA to analyze the RBS region and were compared to the original RBS that corresponds to the strong RBS of pFD119.

Fig. 7. Silencing of $r p s L$ with the optimized vector pFD152 in E. coli. The plasmid carrying the RBS number 4 from the selection process was reprogrammed with a guide RNA carrying BsaI sites and called pFD152. This plasmid was then programmed with guides binding either the E. coli or $S$. aureus rpsL gene on the coding (C-rpsL) or template (T-rpsL) strand. Cells were grown overnight and serial dilutions were performed and plated on LB supplemented with spectinomycin $60 \mu \mathrm{g} / \mathrm{ml}$ in the absence or presence of aTc at $0.5 \mu \mathrm{g} / \mathrm{ml}$. The experiments were performed three times independently.

Fig. 8. Repression of a reporter mCherry by pFD116 or pFD152 as a function of aTc concentration. (A) The $m$ Cherry gene under the control of a constitutive promoter was inserted in plasmids pFD116 and pFD152 expressing dCas9 with a strong and an optimized RBS respectively, giving plasmids pFD165 and pFD166 (Tables 1 and 2). These plasmids were then programmed to target the $m$ Cherry gene with a guide RNA targeting the coding strand (Table 3), and introduced into E. coli MG1655. Serial dilutions were performed from overnight cultures and plated on LB supplemented with spectinomycin $60 \mu \mathrm{g} / \mathrm{ml}$ and in the presence of a range of aTc concentrations. (B) The intensity of mCherry fluorescence from 10 clones was measured and plotted as a function of aTc concentration

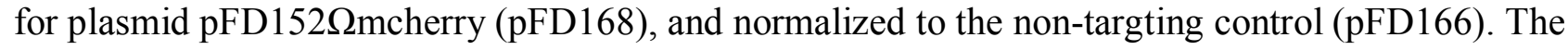
error bars reflect the standard deviation from the mean. 


\section{References}

[1] K.A. Datsenko, B.L. Wanner, One-step inactivation of chromosomal genes in Escherichia coli K-12 using PCR products, P Natl Acad Sci USA. 97 (2000) 6640-6645. doi:10.1073/pnas.120163297.

[2] J.H. Hu, S.M. Miller, M.H. Geurts, W. Tang, L. Chen, N. Sun, C.M. Zeina, X. Gao, H.A. Rees, Z. Lin, D.R. Liu, Evolved Cas9 variants with broad PAM compatibility and high DNA specificity, Nature. 556 (2018) 57-63. doi:10.1038/nature26155.

[3] Y.J. Lee, T.S. Moon, Design rules of synthetic non-coding RNAs in bacteria, Methods. 143 (2018) 58-69. doi:10.1016/j.ymeth.2018.01.001.

[4] Y. Ji, B. Zhang, S.F. Van, null Horn, P. Warren, G. Woodnutt, M.K. Burnham, M. Rosenberg, Identification of critical staphylococcal genes using conditional phenotypes generated by antisense RNA, Science. 293 (2001) 2266-2269. doi:10.1126/science.1063566.

[5] J. Meng, G. Kanzaki, D. Meas, C.K. Lam, H. Crummer, J. Tain, H.H. Xu, A genome-wide inducible phenotypic screen identifies antisense RNA constructs silencing Escherichia coli essential genes, FEMS Microbiol. Lett. 329 (2012) 45-53. doi:10.1111/j.15746968.2012.02503.x.

[6] D. Na, S.M. Yoo, H. Chung, H. Park, J.H. Park, S.Y. Lee, Metabolic engineering of Escherichia coli using synthetic small regulatory RNAs, Nat. Biotechnol. 31 (2013) 170-174. doi:10.1038/nbt.2461.

[7] A.A. Green, P.A. Silver, J.J. Collins, P. Yin, Toehold switches: de-novo-designed regulators of gene expression, Cell. 159 (2014) 925-939. doi:10.1016/j.cell.2014.10.002.

[8] S. Jang, S. Jang, J. Yang, S.W. Seo, G.Y. Jung, RNA-based dynamic genetic controllers: development strategies and applications, Curr. Opin. Biotechnol. 53 (2018) 1-11. doi:10.1016/j.copbio.2017.10.005.

[9] M.N. Win, C.D. Smolke, A modular and extensible RNA-based gene-regulatory platform for engineering cellular function, Proc. Natl. Acad. Sci. U.S.A. 104 (2007) 14283-14288. doi:10.1073/pnas.0703961104.

[10] J.M. Carothers, J.A. Goler, D. Juminaga, J.D. Keasling, Model-driven engineering of RNA devices to quantitatively program gene expression, Science. 334 (2011) 1716-1719. doi:10.1126/science.1212209.

[11] A. Hoynes-O'Connor, T.S. Moon, Development of Design Rules for Reliable Antisense RNA Behavior in E. coli, ACS Synth. Biol. 5 (2016) 1441-1454. doi:10.1021/acssynbio.6b00036.

[12] J. Chappell, M.K. Takahashi, J.B. Lucks, Creating small transcription activating RNAs, Nat. Chem. Biol. 11 (2015) 214-220. doi:10.1038/nchembio.1737.

[13] J.B. Lucks, L. Qi, V.K. Mutalik, D. Wang, A.P. Arkin, Versatile RNA-sensing transcriptional regulators for engineering genetic networks, Proc. Natl. Acad. Sci. U.S.A. 108 (2011) $8617-$ 8622. doi:10.1073/pnas.1015741108.

[14] L.S. Qi, M.H. Larson, L.A. Gilbert, J.A. Doudna, J.S. Weissman, A.P. Arkin, W.A. Lim, Repurposing CRISPR as an RNA-guided platform for sequence-specific control of gene expression, Cell. 152 (2013) 1173-1183. doi:10.1016/j.cell.2013.02.022.

[15] D. Bikard, W. Jiang, P. Samai, A. Hochschild, F. Zhang, L.A. Marraffini, Programmable repression and activation of bacterial gene expression using an engineered CRISPR-Cas system, Nucleic Acids Res. 41 (2013) 7429-7437. doi:10.1093/nar/gkt520.

[16] R. Barrangou, C. Fremaux, H. Deveau, M. Richards, P. Boyaval, S. Moineau, D.A. Romero, P. Horvath, CRISPR provides acquired resistance against viruses in prokaryotes, Science. 315 (2007) 1709-1712. doi:10.1126/science.1138140.

[17] L.A. Marraffini, E.J. Sontheimer, CRISPR interference: RNA-directed adaptive immunity in bacteria and archaea, Nat. Rev. Genet. 11 (2010) 181-190. doi:10.1038/nrg2749.

[18] M. Jinek, K. Chylinski, I. Fonfara, M. Hauer, J.A. Doudna, E. Charpentier, A programmable dual-RNA-guided DNA endonuclease in adaptive bacterial immunity, Science. 337 (2012) 816-821. doi:10.1126/science.1225829. 
[19] K.S. Makarova, Y.I. Wolf, O.S. Alkhnbashi, F. Costa, S.A. Shah, S.J. Saunders, R. Barrangou, S.J.J. Brouns, E. Charpentier, D.H. Haft, P. Horvath, S. Moineau, F.J.M. Mojica, R.M. Terns, M.P. Terns, M.F. White, A.F. Yakunin, R.A. Garrett, J. van der Oost, R. Backofen, E.V. Koonin, An updated evolutionary classification of CRISPR-Cas systems, Nature Reviews Microbiology. 13 (2015) 722-736. doi:10.1038/nrmicro3569.

[20] H. Nishimasu, F.A. Ran, P.D. Hsu, S. Konermann, S.I. Shehata, N. Dohmae, R. Ishitani, F. Zhang, O. Nureki, Crystal Structure of Cas9 in Complex with Guide RNA and Target DNA, Cell. 156 (2014) 935-949. doi:10.1016/j.cell.2014.02.001.

[21] D. Bikard, A. Hatoum-Aslan, D. Mucida, L.A. Marraffini, CRISPR interference can prevent natural transformation and virulence acquisition during in vivo bacterial infection, Cell Host Microbe. 12 (2012) 177-186. doi:10.1016/j.chom.2012.06.003.

[22] D. Bikard, C. Euler, W. Jiang, P.M. Nussenzweig, G.W. Goldberg, X. Duportet, V.A. Fischetti, L.A. Marraffini, Development of sequence-specific antimicrobials based on programmable CRISPR-Cas nucleases, Nat Biotechnol. 32 (2014) 1146-1150. doi:10.1038/nbt.3043.

[23] L. Cui, D. Bikard, Consequences of Cas9 cleavage in the chromosome of Escherichia coli, Nucleic Acids Res. 44 (2016) 4243-4251. doi:10.1093/nar/gkw223.

[24] R.J. Citorik, M. Mimee, T.K. Lu, Sequence-specific antimicrobials using efficiently delivered RNA-guided nucleases, Nature Biotechnology. 32 (2014) 1141-1145. doi:10.1038/nbt.3011.

[25] J.M. Peters, A. Colavin, H. Shi, T.L. Czarny, M.H. Larson, S. Wong, J.S. Hawkins, C.H.S. Lu, B.-M. Koo, E. Marta, A.L. Shiver, E.H. Whitehead, J.S. Weissman, E.D. Brown, L.S. Qi, K.C. Huang, C.A. Gross, A Comprehensive, CRISPR-based Functional Analysis of Essential Genes in Bacteria, Cell. 165 (2016) 1493-1506. doi:10.1016/j.cell.2016.05.003.

[26] X. Liu, C. Gallay, M. Kjos, A. Domenech, J. Slager, S.P. van Kessel, K. Knoops, R.A. Sorg, J.-R. Zhang, J.-W. Veening, High-throughput CRISPRi phenotyping identifies new essential genes in Streptococcus pneumoniae, Mol. Syst. Biol. 13 (2017) 931. doi:10.15252/msb.20167449.

[27] L. Cui, A. Vigouroux, F. Rousset, H. Varet, V. Khanna, D. Bikard, A CRISPRi screen in E. coli reveals sequence-specific toxicity of dCas9, Nat Commun. 9 (2018) 1912. doi:10.1038/s41467-018-04209-5.

[28] F. Rousset, L. Cui, E. Siouve, C. Becavin, F. Depardieu, D. Bikard, Genome-wide CRISPRdCas9 screens in E. coli identify essential genes and phage host factors, PLoS Genet. 14 (2018) e1007749. doi:10.1371/journal.pgen.1007749.

[29] E. Choudhary, P. Thakur, M. Pareek, N. Agarwal, Gene silencing by CRISPR interference in mycobacteria, Nat Commun. 6 (2015) 6267. doi:10.1038/ncomms 7267.

[30] C. Zhao, X. Shu, B. Sun, Construction of a Gene Knockdown System Based on Catalytically Inactive ("Dead") Cas9 (dCas9) in Staphylococcus aureus, Appl. Environ. Microbiol. 83 (2017). doi:10.1128/AEM.00291-17.

[31] A.M. Mariscal, S. Kakizawa, J.Y. Hsu, K. Tanaka, L. González-González, A. Broto, E. Querol, M. Lluch-Senar, C. Piñero-Lambea, L. Sun, P.D. Weyman, K.S. Wise, C. Merryman, G. Tse, A.J. Moore, C.A. Hutchison, H.O. Smith, M. Tomita, J.C. Venter, J.I. Glass, J. Piñol, Y. Suzuki, Tuning Gene Activity by Inducible and Targeted Regulation of Gene Expression in Minimal Bacterial Cells, ACS Synth. Biol. 7 (2018) 1538-1552. doi:10.1021/acssynbio.8b00028.

[32] S.Z. Tan, C.R. Reisch, K.L.J. Prather, A Robust CRISPR Interference Gene Repression System in Pseudomonas, Journal of Bacteriology. 200 (2018) e00575-17. doi:10.1128/JB.00575-17.

[33] T. Wang, C. Guan, J. Guo, B. Liu, Y. Wu, Z. Xie, C. Zhang, X.-H. Xing, Pooled CRISPR interference screening enables genome-scale functional genomics study in bacteria with superior performance, Nat Commun. 9 (2018). doi:10.1038/s41467-018-04899-x.

[34] X. Dong, Y. Jin, D. Ming, B. Li, H. Dong, L. Wang, T. Wang, D. Wang, CRISPR/dCas9mediated inhibition of gene expression in Staphylococcus aureus, J. Microbiol. Methods. 139 (2017) 79-86. doi:10.1016/j.mimet.2017.05.008.

[35] S. Horinouchi, B. Weisblum, Nucleotide sequence and functional map of pC194, a plasmid that specifies inducible chloramphenicol resistance, J. Bacteriol. 150 (1982) 815-825. 
[36] W.M. De Vos, Gene cloning in lactic streptococci., Neth. Milk Dairy J. (1986) 141-154.

[37] J. Perez-Casal, M.G. Caparon, J.R. Scott, Mry, a trans-acting positive regulator of the M protein gene of Streptococcus pyogenes with similarity to the receptor proteins of two-component regulatory systems, J. Bacteriol. 173 (1991) 2617-2624.

[38] A.T. Pulliainen, S. Haataja, S. Kähkönen, J. Finne, Molecular basis of H2O2 resistance mediated by Streptococcal Dpr. Demonstration of the functional involvement of the putative ferroxidase center by site-directed mutagenesis in Streptococcus suis, J. Biol. Chem. 278 (2003) 7996-8005. doi:10.1074/jbc.M210174200.

[39] A.V. Bryksin, I. Matsumura, Rational Design of a Plasmid Origin That Replicates Efficiently in Both Gram-Positive and Gram-Negative Bacteria, PLoS One. 5 (2010). doi:10.1371/journal.pone.0013244.

[40] L.K. Husmann, J.R. Scott, G. Lindahl, L. Stenberg, Expression of the Arp protein, a member of the M protein family, is not sufficient to inhibit phagocytosis of Streptococcus pyogenes, Infect. Immun. 63 (1995) 345-348.

[41] W.M. De Vos, Gene cloning and expression in lactic streptococci, FEMS Microbiology Letters. 46 (1987) 281-295. doi:10.1016/0378-1097(87)90113-3.

[42] R.R. Sanoja, J. Morlon-Guyot, J.P. Guyot, Electrotransformation of Lactobacillus manihotivorans LMG 18010T and LMG 18011, Journal of Applied Microbiology. 87 (1999) 99-107. doi:10.1046/j.1365-2672.1999.00800.x.

[43] I. Biswas, J.K. Jha, N. Fromm, Shuttle expression plasmids for genetic studies in Streptococcus mutans, Microbiology. 154 (2008) 2275-2282. doi:10.1099/mic.0.2008/019265-0.

[44] M.L. Korir, R.A. Flaherty, L.M. Rogers, J.A. Gaddy, D.M. Aronoff, S.D. Manning, Investigation of the Role That NADH Peroxidase Plays in Oxidative Stress Survival in Group B Streptococcus, Front. Microbiol. 9 (2018). doi:10.3389/fmicb.2018.02786.

[45] G. Demarre, A.-M. Guérout, C. Matsumoto-Mashimo, D.A. Rowe-Magnus, P. Marlière, D. Mazel, A new family of mobilizable suicide plasmids based on broad host range R388 plasmid (IncW) and RP4 plasmid (IncPalpha) conjugative machineries and their cognate Escherichia coli host strains, Res. Microbiol. 156 (2005) 245-255. doi:10.1016/j.resmic.2004.09.007.

[46] L. Ferrieres, G. Hemery, T. Nham, A.-M. Guerout, D. Mazel, C. Beloin, J.-M. Ghigo, Silent Mischief: Bacteriophage Mu Insertions Contaminate Products of Escherichia coli Random Mutagenesis Performed Using Suicidal Transposon Delivery Plasmids Mobilized by BroadHost-Range RP4 Conjugative Machinery, Journal of Bacteriology. 192 (2010) 6418-6427. doi:10.1128/JB.00621-10.

[47] J.M. Peters, B.-M. Koo, R. Patino, G.E. Heussler, C.C. Hearne, J. Qu, Y.F. Inclan, J.S. Hawkins, C.H.S. Lu, M.R. Silvis, M.M. Harden, H. Osadnik, J.E. Peters, J.N. Engel, R.J. Dutton, A.D. Grossman, C.A. Gross, O.S. Rosenberg, Enabling genetic analysis of diverse bacteria with Mobile-CRISPRi, Nature Microbiology. 4 (2019) 244. doi:10.1038/s41564-0180327-z.

[48] C. Engler, R. Kandzia, S. Marillonnet, A One Pot, One Step, Precision Cloning Method with High Throughput Capability, PLoS ONE. 3 (2008) e3647. doi:10.1371/journal.pone.0003647.

[49] B.N. Kreiswirth, S. Löfdahl, M.J. Betley, M. O'Reilly, P.M. Schlievert, M.S. Bergdoll, R.P. Novick, The toxic shock syndrome exotoxin structural gene is not detectably transmitted by a prophage, Nature. 305 (1983) 709-712.

[50] E.A. Boyle, J.O.L. Andreasson, L.M. Chircus, S.H. Sternberg, M.J. Wu, C.K. Guegler, J.A. Doudna, W.J. Greenleaf, High-throughput biochemical profiling reveals sequence determinants of dCas9 off-target binding and unbinding, Proc Natl Acad Sci USA. 114 (2017) 5461-5466. doi:10.1073/pnas.1700557114.

[51] R.T. Leenay, K.R. Maksimchuk, R.A. Slotkowski, R.N. Agrawal, A.A. Gomaa, A.E. Briner, R. Barrangou, C.L. Beisel, Identifying and Visualizing Functional PAM Diversity across CRISPR-Cas Systems, Molecular Cell. 62 (2016) 137-147. doi:10.1016/j.molcel.2016.02.031.

[52] R. Green, E.J. Rogers, Transformation of Chemically Competent E. coli, in: Methods in Enzymology, Elsevier, 2013: pp. 329-336. doi:10.1016/B978-0-12-418687-3.00028-8. 
[53] R. Novick, Properties of a cryptic high-frequency transducing phage in Staphylococcus aureus, Virology. 33 (1967) 155-166. doi:10.1016/0042-6822(67)90105-5.

[54] I.R. Monk, J.J. Tree, B.P. Howden, T.P. Stinear, T.J. Foster, Complete Bypass of Restriction Systems for Major Staphylococcus aureus Lineages, MBio. 6 (2015) e00308-15. doi:10.1128/mBio.00308-15.

[55] M. Pagels, S. Fuchs, J. Pané-Farré, C. Kohler, L. Menschner, M. Hecker, P.J. McNamarra, M.C. Bauer, C. von Wachenfeldt, M. Liebeke, M. Lalk, G. Sander, C. von Eiff, R.A. Proctor, S. Engelmann, Redox sensing by a Rex-family repressor is involved in the regulation of anaerobic gene expression in Staphylococcus aureus, Mol. Microbiol. 76 (2010) 1142-1161. doi:10.1111/j.1365-2958.2010.07105.x.

[56] E.M. Ozbudak, M. Thattai, I. Kurtser, A.D. Grossman, A. van Oudenaarden, Regulation of noise in the expression of a single gene, Nat Genet. 31 (2002) 69-73. doi:10.1038/ng869.

[57] J. Paulsson, Models of stochastic gene expression, Physics of Life Reviews. 2 (2005) 157-175. doi:10.1016/j.plrev.2005.03.003.

[58] D.G. Gibson, L. Young, R.-Y. Chuang, J.C. Venter, C.A. Hutchison, H.O. Smith, Enzymatic assembly of DNA molecules up to several hundred kilobases, Nat. Methods. 6 (2009) 343-345. doi:10.1038/nmeth.1318.

[59] F. St-Pierre, L. Cui, D.G. Priest, D. Endy, I.B. Dodd, K.E. Shearwin, One-step cloning and chromosomal integration of DNA, ACS Synth Biol. 2 (2013) 537-541. doi:10.1021/sb400021j.

[60] H.M. Salis, E.A. Mirsky, C.A. Voigt, Automated design of synthetic ribosome binding sites to control protein expression, Nature Biotechnology. 27 (2009) 946-950. doi:10.1038/nbt.1568.

[61] I. Farasat, M. Kushwaha, J. Collens, M. Easterbrook, M. Guido, H.M. Salis, Efficient search, mapping, and optimization of multi-protein genetic systems in diverse bacteria, Molecular Systems Biology. 10 (2014) 731. doi:10.15252/msb.20134955.

[62] F. Ceroni, R. Algar, G.-B. Stan, T. Ellis, Quantifying cellular capacity identifies gene expression designs with reduced burden, Nat Methods. 12 (2015) 415-418. doi:10.1038/nmeth.3339. 


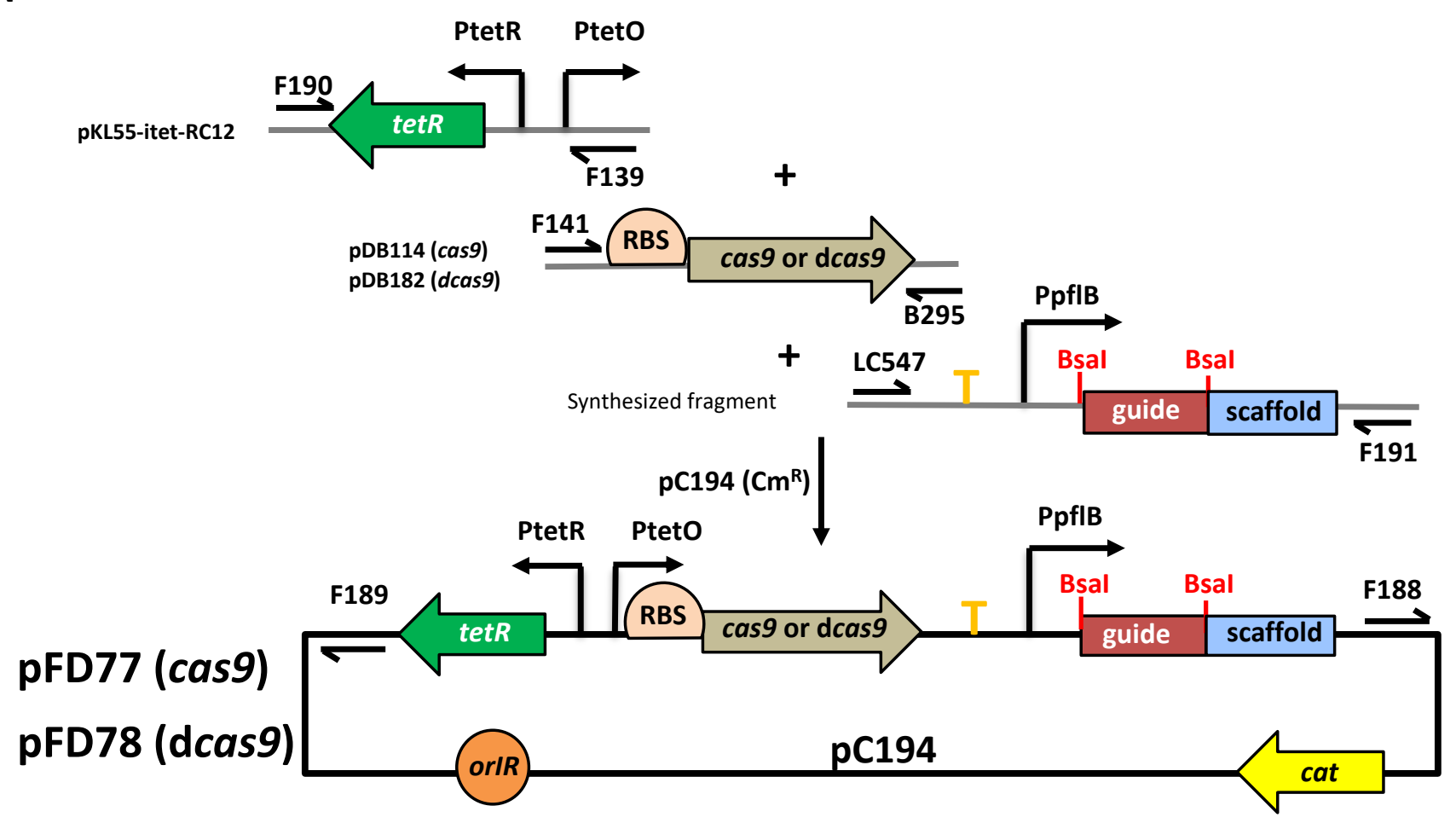

B

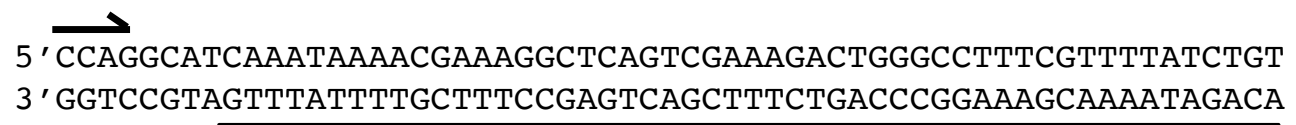
rrnB T1 terminator

5 ' TGTTTGTCGGTGAACGCTCTCTACTAGAGTCACACTGGCTCACCTTCGGGTGGGCCTTTCTGCGTTTATA 3' ACAAACAGCCACTTGCGAGAGATGATCTCAGTGTGACCGAGTGGAAGCCCACCCGGAAAGACGCAAATAT rrnB T1 terminator T7Te terminator

5 ' ATATACTCCTAAATTAACTTTTAAAGCAATGAAAATAGTGAACATTATAACTGTTGTGTAACAGAATGCA 3' TATATGAGGATTTAATTGAAAATTTCGTTACTTTTATCACTTGTAATATTGACAACACATTGTCTTACGT

\section{PpflB promoter}

5' ATTAGCATATTACTGTTACACAAATTAGTACAGTTTCTATGTTTTGACATACATTTGATGAAAATTGTAC 3' TAATCGTATAATGACAATGTGTTTAATCATGTCAAAGATACAAAACTGTATGTAAACTACTTTTAACATG

\section{PpflB promoter}

Bsal

5 ' ATAATTTATGTGAAAAAAATCACAACAAACATGCTACAATCTAATGAGACCAGTCTAGGTCTCGGTTTTA 3' TATTAAATACACTTTTTTTAGTGTTGTTTGTACGATGTTAGATTACTCTGGTCAGATCCAGAGCCAAA AT PpflB promoter ${ }_{4}$ Bsal guice

5' GAGCTAGAAATAGCAAGTTAAAATAAGGCTAGTCCGTTATCAACTTGAAAAAGTGGCACCGAGTCGGTGC 3' CTCGATCTTTATCGTTCAATTTTATTCCGATCAGGCAATAGTTGAACTTTTTCACCGTGGCTCAGCCACG

\section{scaffold}

5' TTTTTTTGGAGATCTGTCCATACCCATGGATTCTTCGTCTGTTTCTACTCCATCAGTCTGACGA

3' AAAAAAACCTCTAGACAGGTATGGGTACCTAAGAAGCAGACAAAGATGAGGTAGTCAGACTGCT

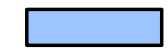




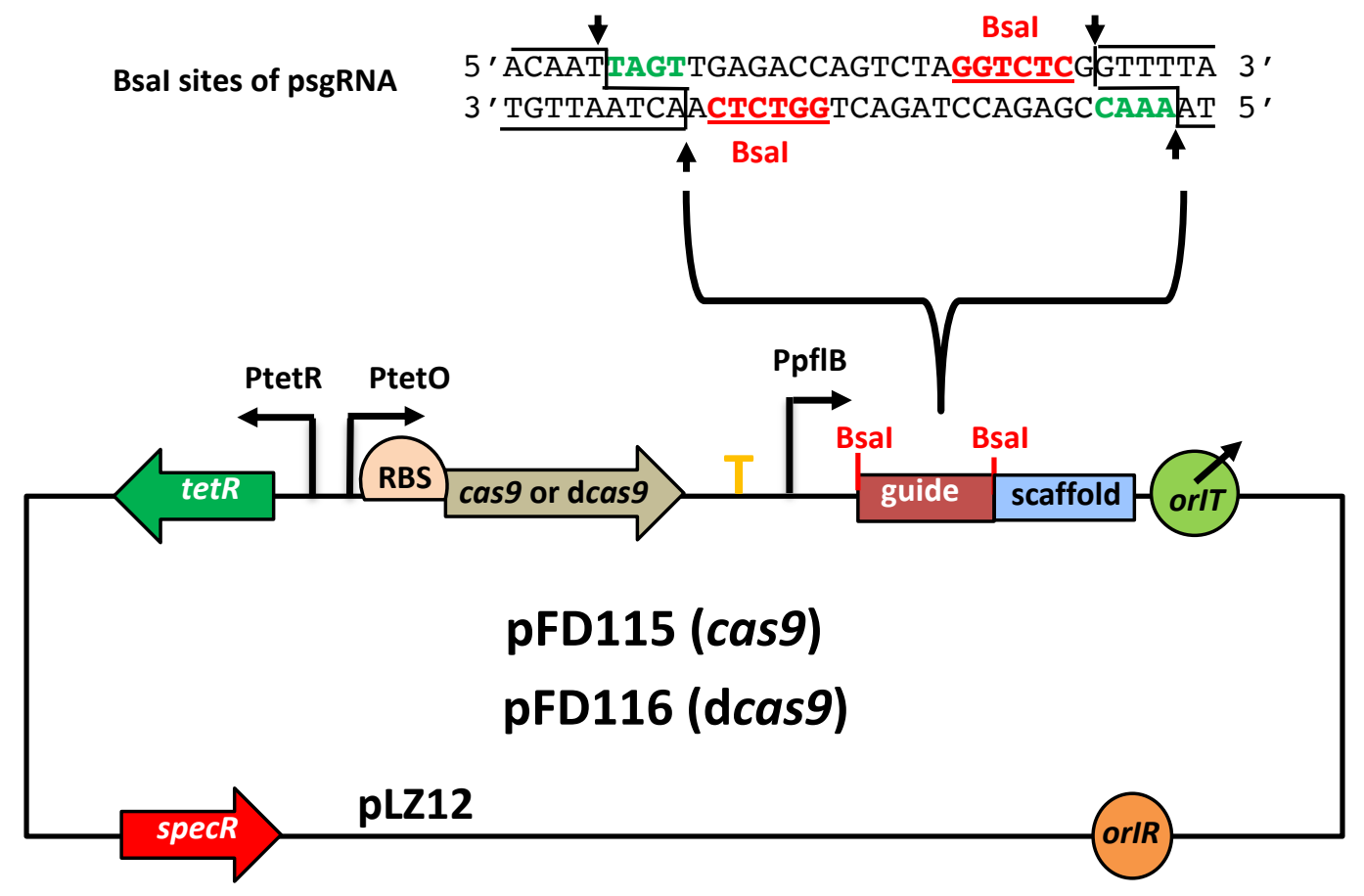

Fig.2 


\section{Target selection}

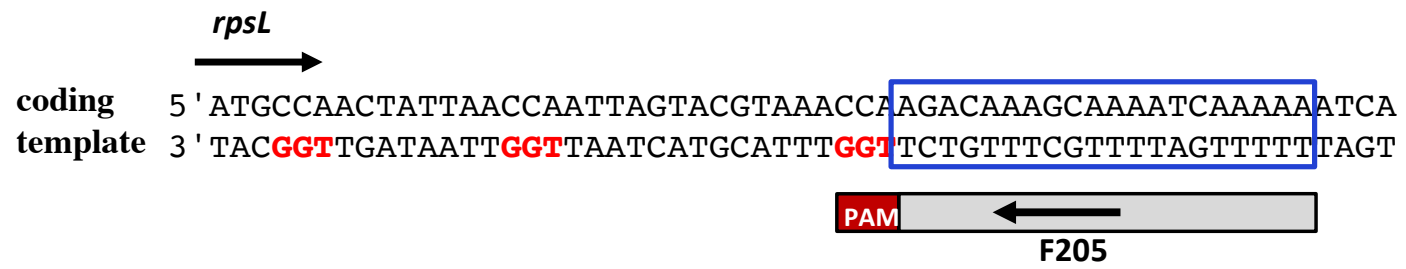

coding 5'GATTCTCCAGCTTTAAATAAAGGTTTCAA--n-

template 3 ' CTAAGAGGTCGAAATTTATTTCCAAAGTT=--

\section{Synthesis of oligonucleotides}

$$
\begin{array}{lll}
\text { Forward primer F205 5'-CTAATTTTTGATTTTGCTTTGTCT -3' } \\
\text { Reverse primer F153 5' } 5 \text {-AAACAGACAAAGCAAAATCAAAAA -3' }
\end{array}
$$

\section{Phosphorylation and annealing of oligonucleotides}

$$
\begin{aligned}
& \text { 5' P-CTAATTTTTGATTTTGCTTTGTCT-3' } \\
& \text { 3'-AAAAACTAAAACGAAACAGACAAA-P 5' }
\end{aligned}
$$

4. Cloning of oligonucleotides by Golden Gate method

$5^{\prime}$ - CAAACATGCTACAATCTAATGAGACCAGTCTAGGTCTCGGTTTTAGAGCTAGAAA-3 '

3' -GTTTGTACGATGTTAGATTACTCTGGTCAGATCCAGAGCCAAA ATCTCGATCTTT-5'

$$
4 \text { Bsal }
$$

Digestion of plasmid by BsaI

$$
\begin{aligned}
& \text { 5'-CAAACATGCTACAAT } \\
& \text { 3'-GTTTGTACGATGTTAGATT }
\end{aligned}
$$

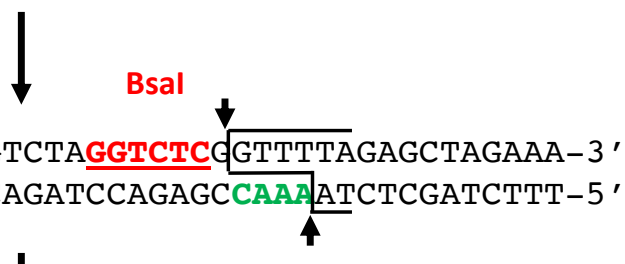

$\downarrow$

\section{ed primers}

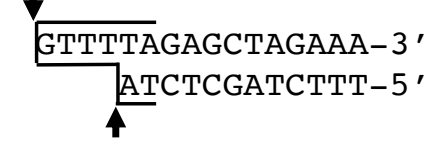

Cloning of phosphorylated and annealed primers

5' -CAAACATGCTACAATCTAATTTTTGATTTTGCTTTGTCTGTTTTAGAGCTAGAAA-3'

3' -GTTTGTACGATGTTAGATTAAAAACTAAAACGAAACAGACAAAATCTCGATCTTT-5'

5. Dialysis at room temperature for $\mathbf{2 0} \mathrm{min}$

6. Transformation

Fig.3 
A

S. aureus RN4220/

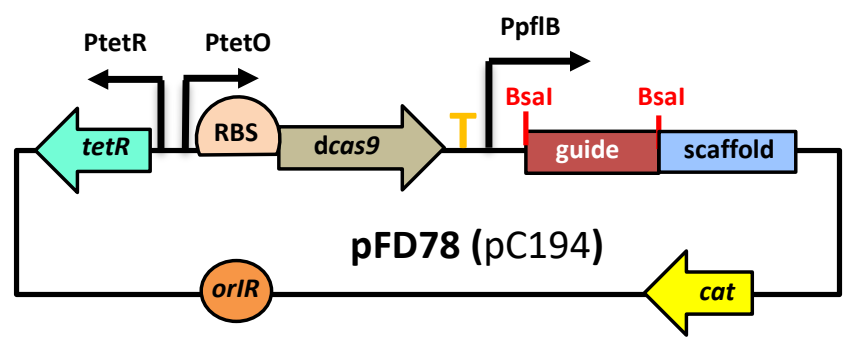

not induced

empty vector control sgRNA

T-rpsL

C-rpsL

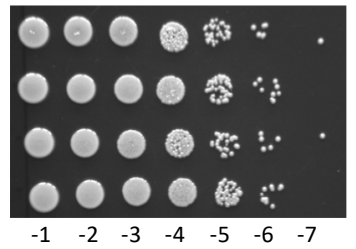

Log 10 dilution

+ aTc $0.025 \mu \mathrm{g} / \mathrm{ml}$

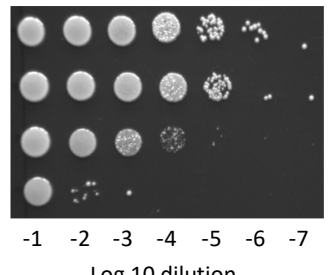

C

S. aureus RN4220/pFD77 (pC194 $\Omega$ cas9)

not induced

+ aTc $0.025 \mu \mathrm{g} / \mathrm{ml}$

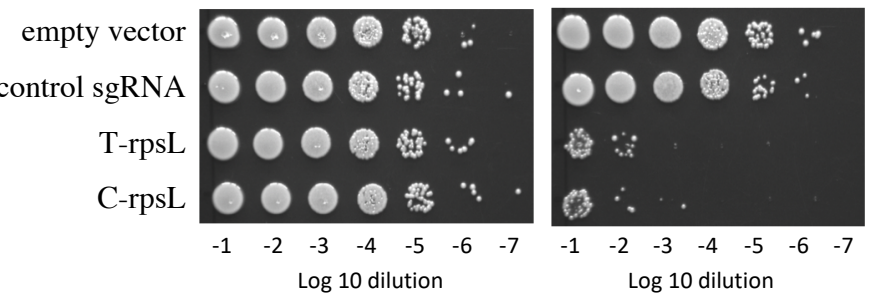

B

S. aureus RN4220/

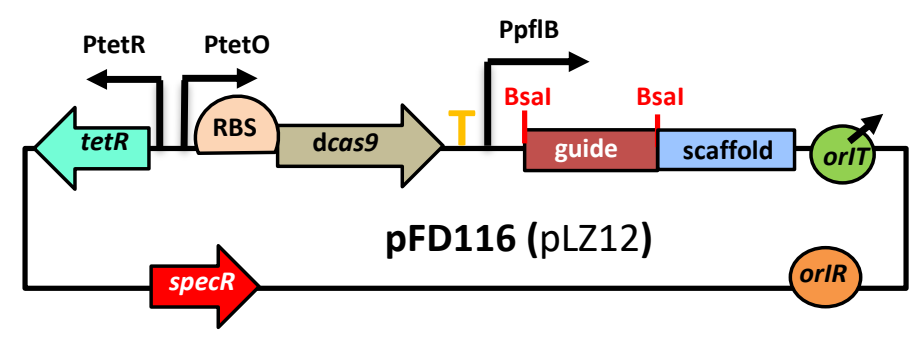

not induced

+ aTc $0.5 \mu \mathrm{g} / \mathrm{ml}$

empty vector $\mathrm{O} O \mathrm{O} 6 \mathrm{6}$ control sgRNA $\bigcirc \bigcirc \bigcirc$

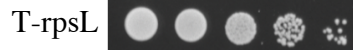
C-rpsL $O 00$ 解 ACCCA $О 00 \bigcirc \div$ AGGAA $\bigcirc \bigcirc \bigcirc \bigcirc$

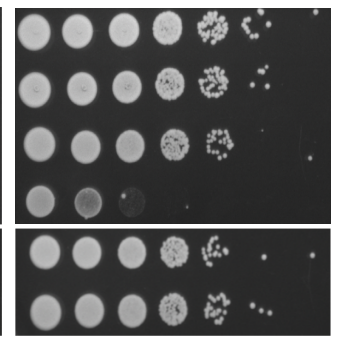

$\begin{array}{lllll}-1 & -2 & -3 & -4 & -5\end{array}$

$\begin{array}{lllllll}-1 & -2 & -3 & -4 & -5 & -6 & -7\end{array}$ Log 10 dilution

D

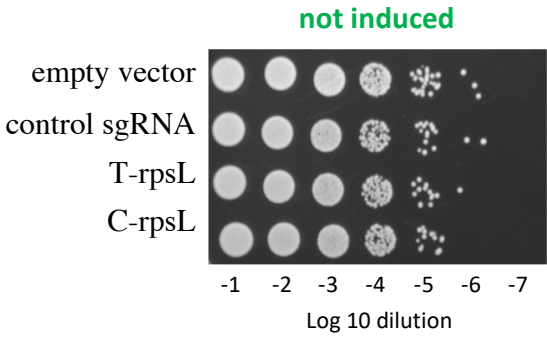

+ aTc $0.5 \mu \mathrm{g} / \mathrm{ml}$

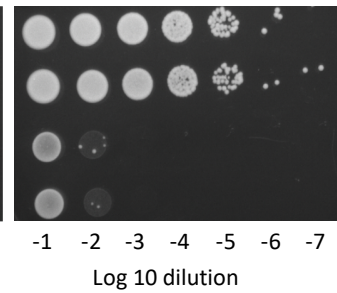

Fig. 4 
A

S. aureus $8325-4$ /

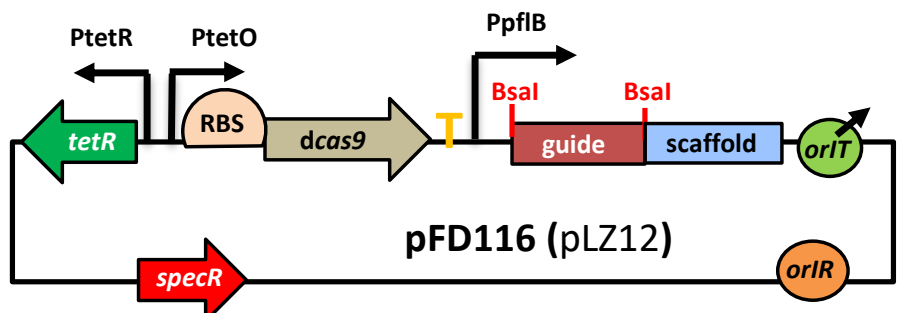

not induced

empty vector

0000 :

$$
\text { T-rpsL }
$$

O000

C-rpsL

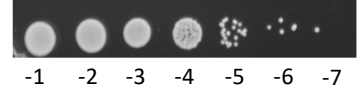

Log 10 dilution
B

S. aureus $\mathbf{8 3 2 5 - 4 /}$
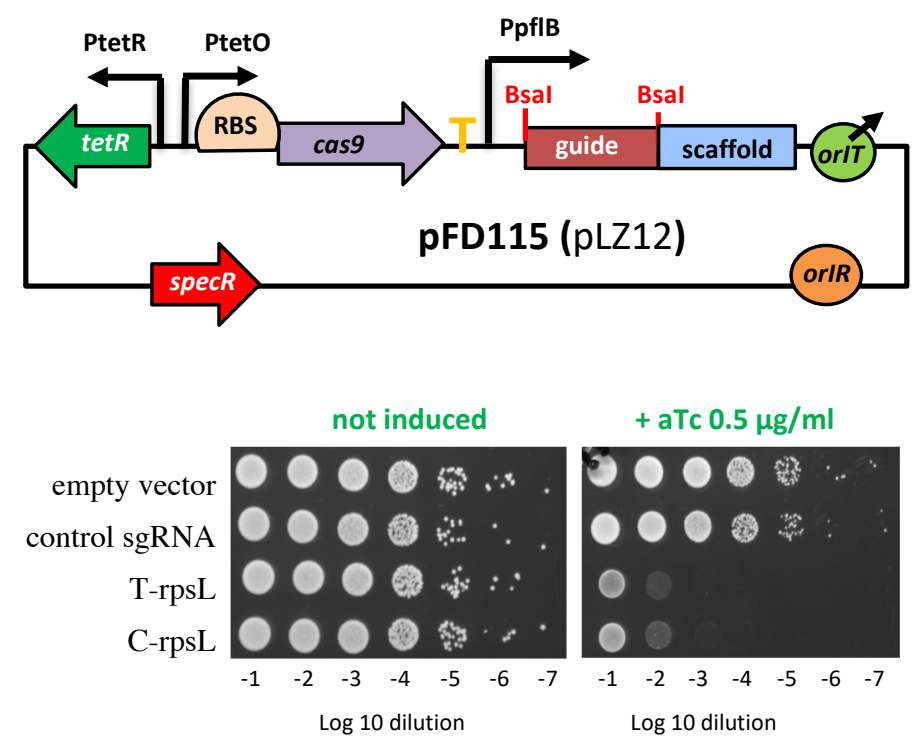

Fig. 5 
1. Insertion of RBS library with primers F308/F309 by Gibson assembly
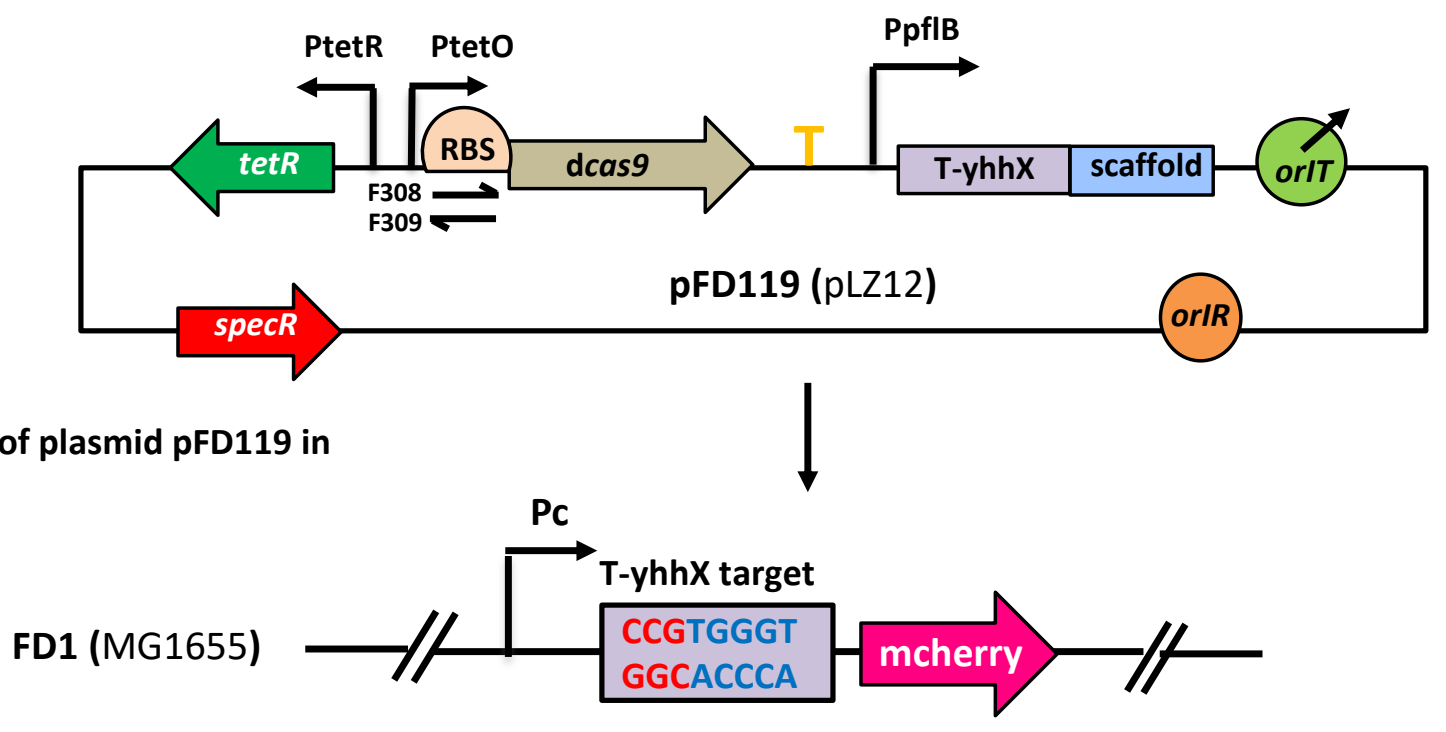

\section{Selection on LB +}

$$
\begin{aligned}
& \operatorname{Spc} 60 \mu \mathrm{g} / \mathrm{ml} \\
& +\operatorname{aTc} 0.5 \mu \mathrm{g} / \mathrm{ml}
\end{aligned}
$$
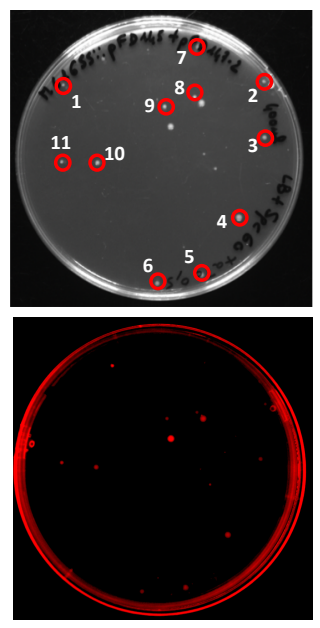

4. Screen of clones with non toxic expression levels of dCas9 and good repression of mcherry with aTc

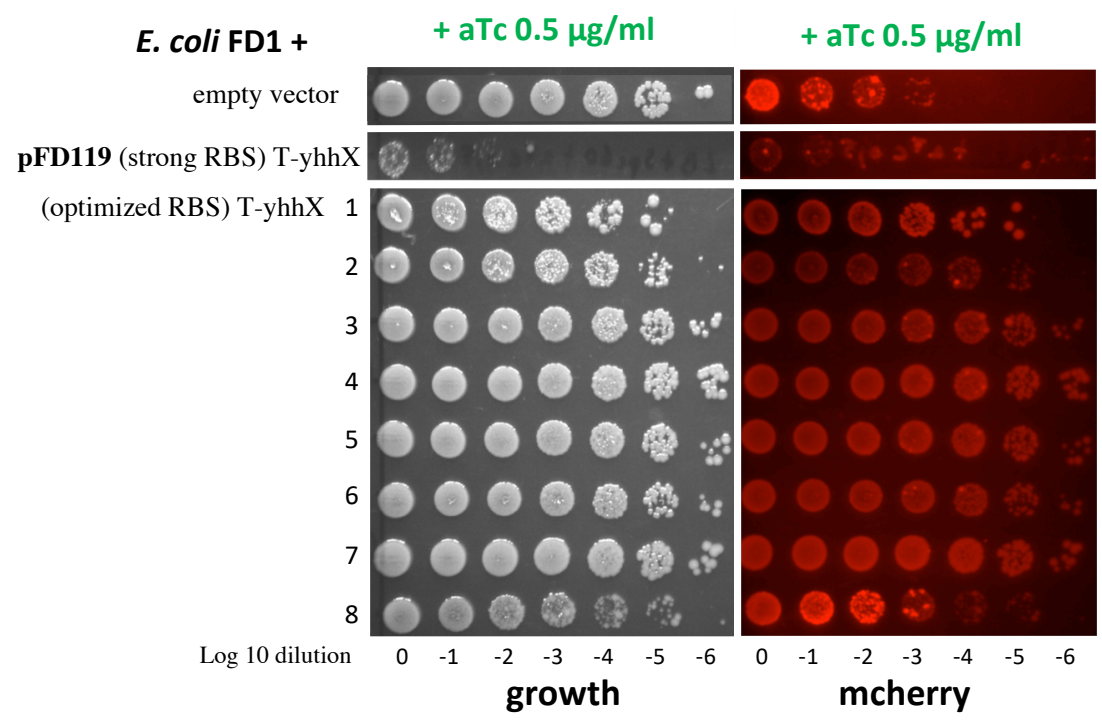

\section{Sequencing of the RBS}

using a spectrophotometer

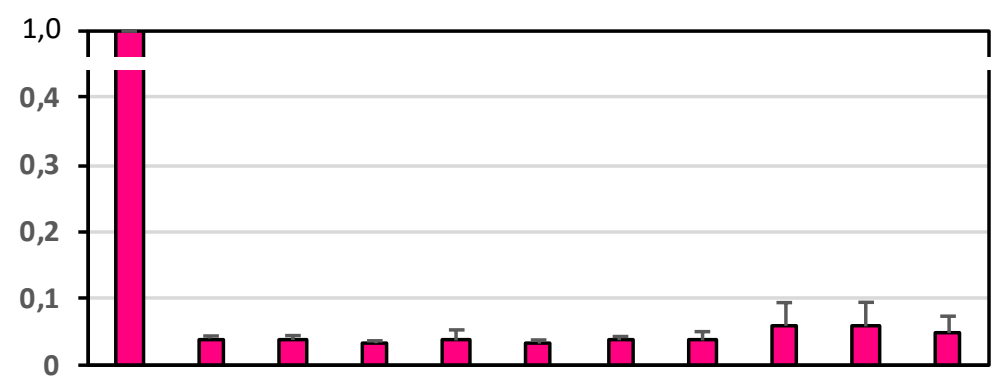

pLZ12 Clone Clone Clone Clone Clone Clone Clone Clone Clone Clone

$\begin{array}{llllllllll}4 & 5 & 7 & 12 & 13 & 15 & 16 & 18 & 14 & 17\end{array}$

FD1

FD1+ pFD119-RBS-library
pFD119 TATCGAATTCGGAGGCATTATAGAGGCAAAA RBS $\mathbf{l i b}$ TATCGAATTCNGANGCATNNNNGNNNCAAAA

clone 4 TATCGAATTCGGACGCATAGTGGCCTCAAAA clone 5 TATCGAATTCCGATGCATCGTGGCAGCAAAA clone 7 TATCGAATTCGGACGCATGCTAGCGGCAAAA clone 12 TATCGAATTCCGAGGCATGCTCGTGTCAAAA clone 13 TATCGAATTCGGACGCATGCGGGCCCCAAAA Clone 15 TATCGAATTCTGATGCATCCGTGTTGCAAAA clone 16 TATCGAATTCTGATGCATCCGTGTTGCAAAA clone 18 TATCGAATTCCGACGCATCTGGGCTACAAAA clone 14 TATCGAATTCCGACGCATCGG-GTGTCAAAA clone 17 TA----ATTCCGAAGCATCTCGGCCGCAAAA

Fig. 6 


\section{E. coli MG1655}

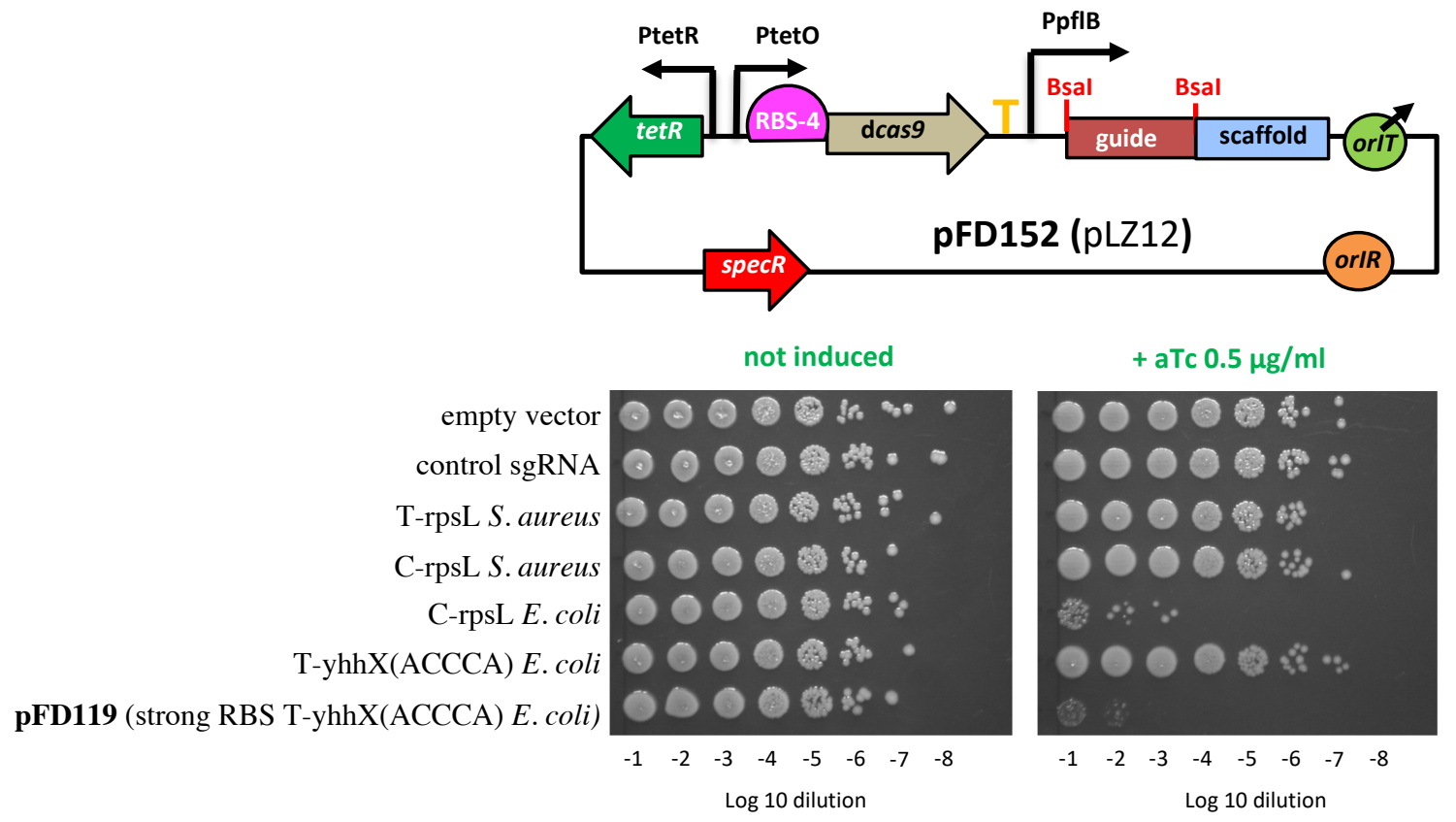

Fig. 7 
A

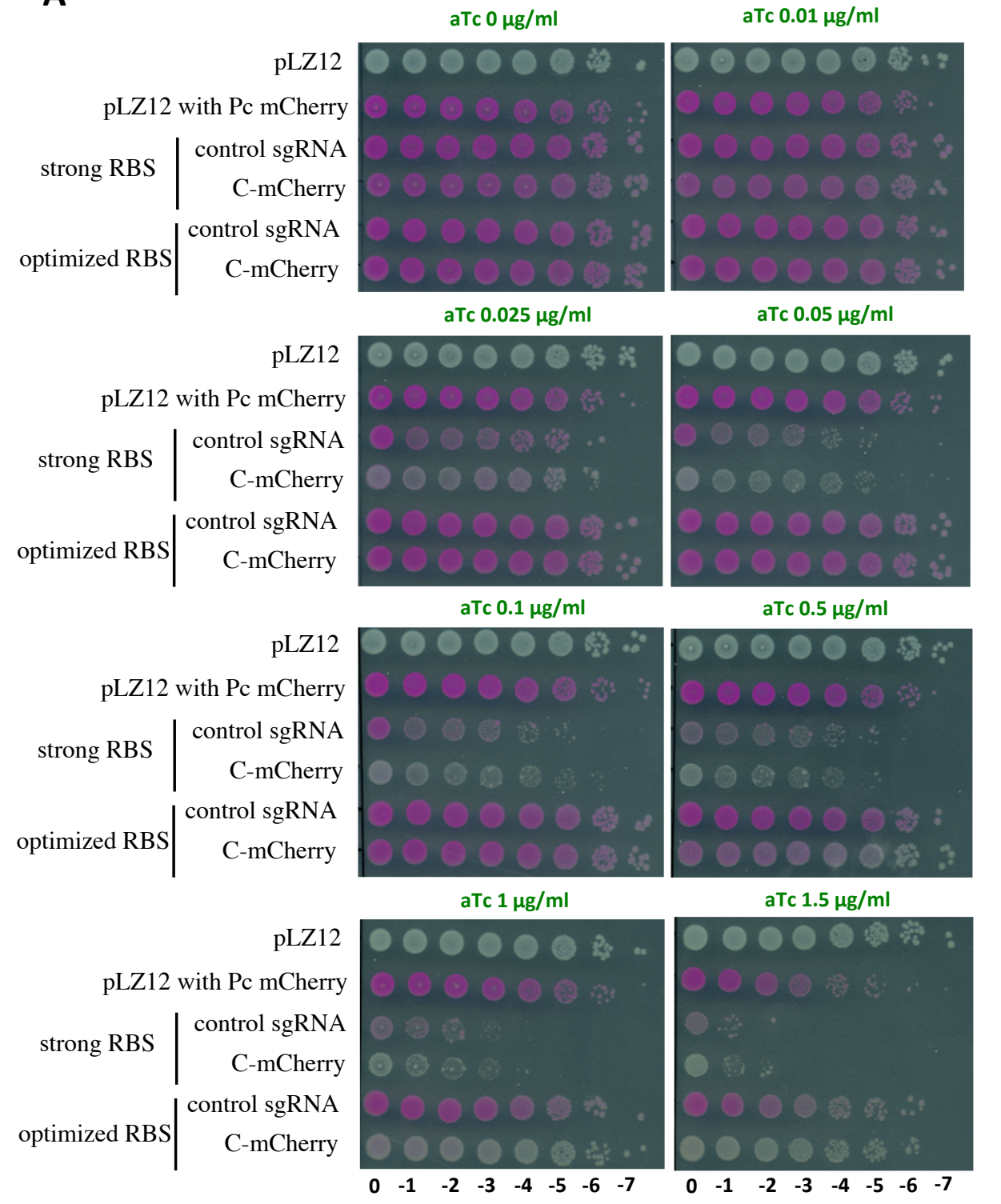

B

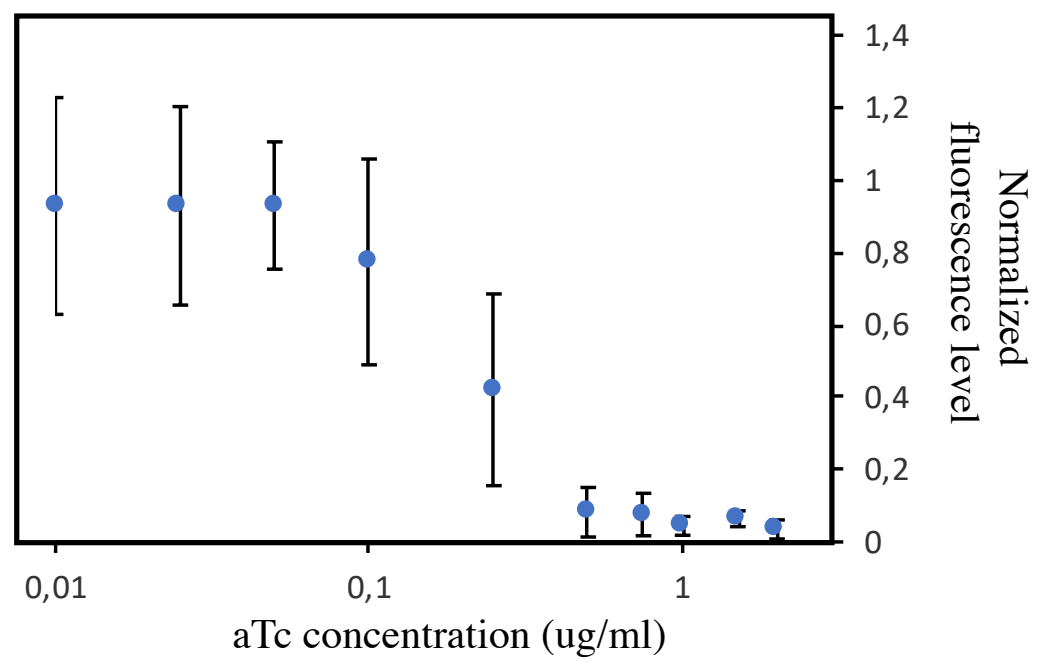

Fig.8 